\title{
Potential Theory of Hyperfinite Dirichlet Forms*
}

\author{
RU-ZONG FAN \\ Department of Probability and Statistics, Peking University, Beijing 10987I, P.R. China; and \\ Institute of Mathematics, Ruhr University Bochum, Postfach 102148, D-44780 Bochum 1, Germany \\ (Current address: Department of Biostatistics, University of Michigan, Ann Arbor, MI 48109, U.S.A. \\ e-mail: rfan@sph.umich.edu)
}

(Received: 28 September 1993; accepted: 22 December 1994)

\begin{abstract}
In this paper, we are going to study the capacity theory and exceptionality of hyperfinite Dirichlet forms. We shall introduce positive measures of hyperfinite energy integrals and associated theory. Fukushima's decomposition theorem will be established on the basis of discussing hyperfinite additive functionals and hyperfinite measures. We shall study the properties of internal multiplicative functionals, subordinate semigroups and subprocesses. Moreover, we shall discuss transformation of hyperfinite Dirichlet forms.
\end{abstract}

Mathematics Subject Classifications (1991). Primary 60J25, 60J45, 60J57, 60J60; Secondary $03 \mathrm{H} 05,03 \mathrm{H} 10,31 \mathrm{C} 15,31 \mathrm{C} 25$.

Key words: Additive functional, capacity theory, Dirichlet form, energy, exceptionality, generator, Markov chain, measure of hyperfinite energy integral, multiplicative functional, patential, semi-group. subordinate semigroup and subprocess.

\section{Introduction}

Probabilistic potential theory has been a very important part in the study of standard Dirichlet space theory. It establishes a beautiful bridge between functional analysis and the theory of symmetric Markov processes. It gives us probabilistic interpretation of potential theory. Many applications of this theory have been found, especially in the area of mathematical physics. Our purpose in this paper is to develop potential theory associated with the hyperfinite Dirichlet forms. The motivation is twofold. On the one hand, we would like to establish a relationship between the standard Dirichlet space theory and the hyperfinite counterpart. On the other hand, we are interested in the theory of hyperfinite Dirichlet forms itself. We hope to construct the completed theory system for hyperfinite Dirichlet space theory. Besides, we have studied infinite dimensional stochastic analysis extensively in recent years. It is our hope to find some new and powerful machinery to deal with the problems in this exciting area. Maybe the nonstandard analysis is one of these methods. In fact, we have utilized our theory of hyperfinite Dirichlet forms in the study of [6], in which the important construction of strong Markov

* Research was supported by the National Natural Science Foundation of P.R. China, No. 18901004. The support from the position of Wissenschaftliche Hilfskraft of Ruhr-University Bochum under Prof. Sergio Albeverio is also acknowledged. 
processes associated with quasi-regular Dirichlet forms has been fulfilled by using the nonstandard analysis language. For another method of construction, the reader can refer to [4].

The study of hyperfinite Dirichlet space theory was initiated by [3]. First of all, the authors introduced the frameworks of hyperfinite Dirichlet forms. When the state space is a hyperfinite subset $S_{0}$ of ${ }^{*} Y$ for some Hausdorff topological space $Y$, they established an one to one correspondence between the family of hyperfinite Dirichlet forms and the family of symmetric Markov chains. Furthermore, they obtained hyperfinite lifting $\mathcal{E}(\cdot, \cdot)$ for every standard Dirichlet form $E(\cdot, \cdot)$. The standard parts of hyperfinite Markov chains have been studied in the work also. In fact, some aspects of our potential theory have been considered by the authors, such as exceptional sets, Feynman-Kac formulae, equilibrium potentials. However, we shall study potential theory of hyperfinite Dirichlet forms systematically in this paper. New concept and method will be presented as the paper develops.

Now let us talk about the arrangement of this paper. In Section 2, we shall introduce the framework of hyperfinite Markov chains and associated Dirichlet forms. The authors of [3] have given us a definition of exceptional sets, which might be too restrictive for certain case. Therefore, we define this concept in a new way in Section 3. Moreover, we introduce capacity for the hyperfinite Dirichlet forms and show that it is a Choquet capacity. Furthermore, we establish a relationship between the family of exceptional sets and the family of zero capacity sets. In Section 4, we consider positive measures of hyperfinite energy integrals and associated theory, that is, the connections among hyperfinite excessive functions and hyperfinite potentials. The zero capacity subsets will be characterized by the language of positive measures of hyperfinite energy integrals. In Section 5, we introduce internal additive functionals. The relationship between hyperfinite measures and additive functionals will be considered. Moreover, we obtain the positive hyperfinite measure $\mu_{\langle u\rangle}(i)$ associated with an internal function $u$. In Section 6, we get the Fukushima's decomposition theorem under the individual probability measures $P_{i}, i \in S_{0}$. This extends the work of [3], since they have completed the decomposition under the whole measure $P_{m}$. In Section 7 , we will discuss the properties of internal multiplicative functionals, subordinate semigroups, subprocesses and a Feynman-Kac formula. The motivation of this work is the similar standard theory developed by [5]. Moreover, we hope to study perturbation theory of the hyperfinite Dirichlet forms. In fact, a subject for our future research is the hyperfinite perturbation theory characterized by the language of internal additive functionals. In standard Dirichlet space theory, we have been interested in such question: could we change a non-conservative symmetric Markov process into a conservative one? [8] answered this problem by using Girsanov transformation. In Section 8, we show that this problem is quite simple in hyperfinite settings (Theorem 8.1).

It is well known that in the standard Markov process theory, we can study potential theory in the case of non-symmetry (refer to [5]). In fact, the symmetric condition (2.3) in Section 2 is not necessary in the Definition 3.1 and Definition 3.2 
of exceptional sets. Moreover, we can prove Lemma 3.1 and Proposition 3.1 without this symmetric condition. Besides, we can define additive functionals, hyperfinite excessive functions and multiplicative functionals in the circumstance of nonsymmetry. For the work of non-symmetry, the reader can refer to the book [1] in preparation and paper [2], also [9]. Nevertheless, let us work under the symmetric situation since in most part of this paper, it is still necessary.

\section{Hyperfinite Markov Chain and Dirichlet Form}

Let $Y$ be a Hausdorff space and ${ }^{*} Y$ be the nonstandard extension of $Y$. Let $S=\left\{s_{0}, s_{1}, \ldots, s_{N}\right\}$ be an $S$-dense subset of ${ }^{*} Y$ for some $N \in \mathbb{N}-\mathbf{N}$ and $m$ be a hyperfinite measure on $S$. Denote by $\mathcal{S}$ the internal algebra of subsets of $S$. We would remind the readers here the difference between $S$ and $\mathcal{S}$. Assume that $Q=\left\{q_{i j}\right\}$ is an $(N+1) \times(N+1)$ matrix with non-negative entries, and assume that

$$
\sum_{j=0}^{N} q_{i j}=1 \text { for all } i \in S,
$$

and the state $s_{0}$ is a trap, i.e.,

$$
q_{0 i}=0 \text { for all } i \neq 0 \text {. }
$$

In the sequel, we shall write $m_{i}$ for $m\left(\left\{s_{i}\right\}\right)$ and $q_{i j}$ for $q_{s_{i} s_{j}}$ respectively, whenever it is convenient.

We assume the measure $m$ and the transition matrix $Q$ satisfy symmetry conditions

$$
m_{i} q_{i j}=m_{j} q_{j i} \quad \text { for all } i \neq 0, j \neq 0 .
$$

Besides, we assume that

$$
m_{i} \neq 0 \text { for at least one } i \neq 0 \text {. }
$$

Take an infinitesimal $\Delta t$ such that $0<\Delta t$. Set

$$
T=\left\{k \Delta t \mid k \in \in^{*} \mathbf{N}\right\} \text {. }
$$

If $(\Omega, P)$ is an internal measure space, and $X: \Omega \times T \rightarrow S$ is an internal process, let

$$
[\omega]_{t}=\left\{\omega^{\prime} \in \Omega \mid X\left(\omega^{\prime}, s\right)=X(\omega, s) \text { for all } s \leqslant t\right\} .
$$

For each $t \in T$, let $\mathcal{F}_{t}$ be the internal algebra on $\Omega$ generated by all sets $[\omega]_{t}$. Assume that for all $\omega$

$$
P\left(\{\omega]_{0}\right)=m\{X(\omega, 0)\}
$$


and whenever $X(\omega, t)=s_{i}$,

$$
P\left\{\omega^{\prime} \in[\omega]_{t} \mid X\left(t+\Delta t, \omega^{\prime}\right)=s_{j}\right\}=q_{i j} P\left([\omega]_{t}\right) .
$$

In particular, we define a family $\left(\Omega, \mathcal{F}_{t}, P_{i}, i \in S\right)$ of internal probability spaces by

$$
P_{i}\left([\omega]_{k \Delta t}\right)=\delta_{i \omega(0)} \prod_{n=0}^{k-1} q_{\omega(n \Delta t), \omega((n+1) \Delta t)}
$$

for each $i \in S$, where $\delta_{i j}$ is the kronecker symbol.

A process $X$ satisfying (2.1), (2.2), (2.3), (2.4), (2.7) and (2.8) is called a symmetric hyperfinite Markov chain associated with $m$ and $Q$, and it is this kind of processes we will study in detail. We shall first obtain the form from the process.

If

$$
S_{0}=\left\{s_{1}, s_{2}, \ldots, s_{N}\right\}
$$

is the state space $S$ without the trap $s_{p}$, denote $\mathcal{S}_{0}=\mathcal{S} \cap S_{0}$. Let $H$ be the linear space of all internal functions $u: S_{0} \rightarrow{ }^{*} \mathrm{R}$ with the inner product

$$
\int_{S_{0}} u v \mathrm{~d} m=\langle u, v\rangle=\sum_{i=1}^{N} u\left(s_{i}\right) v\left(s_{i}\right) m\left(s_{i}\right) .
$$

Just as we usually write $m_{i}$ for $m\left(s_{i}\right)$, we shall write $u(i)$ or $u_{i}$ for $u\left(s_{i}\right)$. And we shall identify $H$ with the set of all internal function $u: S \rightarrow{ }^{*} \mathbf{R}$ such that $u\left(s_{0}\right)=0$.

Our convention of letting the trap $s_{0}$ be the zeroth element is notationally convenient, but we call attention of the reader to the fact that he should distinguish between sums of the forms $\Sigma_{i=0}^{N}$ and $\Sigma_{i=1}^{N}$.

For $t \in T$ and $u \in H$, we define a new function $Q^{t} u \in H$ by

$$
Q^{i} u(i)=E_{i} u(X(t)),
$$

where $E_{i}$ is the expectation with respect to the measure $P_{i}$ defined in (2.9). Intuitively, $Q^{t} u(i)$ is the expected value of $u(X(t))$ for a particle starting in state $s_{i}$. Notice that

$$
Q^{\Delta t} u(i)=(Q \cdot u)(i)=\sum_{j=1}^{N} u(j) q_{i j}
$$

where $\cdot$ is the matrix multiplication in the middle term, and since $q_{i j}^{(t+s)}=$ $\Sigma_{k=1}^{N} q_{i k}^{(t)} q_{k j}^{(s)}$, we must have

$$
Q^{t+s}=Q^{t} \cdot Q^{s}
$$


where $q_{i j}^{(t)}$ is the transition probability given by operator $Q^{t}$. Hence the family $\left\{Q^{t} \mid t \in T\right\}$ is a semigroup of operators on $H$.

The infinitesimal generator $A$ of this semigroup is given by

$$
A u(i)=\frac{1}{\Delta t}\left(u(i)-\sum_{j=1}^{N} u(j) q_{i j}\right) .
$$

The Dirichlet form associated with $Q$ and $m$ is defined to be

$$
\mathcal{E}(u, v)=\langle A u, v\rangle=\sum_{i=1}^{N} A u(i) v(i) m(i) .
$$

Combining (2.15) and (2.16), we get

$$
\mathcal{E}(u, v)=\frac{1}{\Delta t} \sum_{i=1}^{N}\left[u(i) v(i) m(i)-\sum_{j=1}^{N} u(j) v(i) q_{i j} m(i)\right] .
$$

Moreover, we have the following Beurling-Deny formula (refer to [3, 5.3.1 Lemma]):

$$
\begin{gathered}
\mathcal{E}(u, v)=\frac{1}{\Delta t}\left[\sum_{1 \leqslant i<j \leqslant N}(u(i)-u(j))(v(i)-v(j)) q_{i j} m(i)\right. \\
\left.+\sum_{i=1}^{N} u(i) v(i) q_{i 0} m(i)\right]
\end{gathered}
$$

The domain $\mathcal{D}(\mathcal{E})$ of $\mathcal{E}(\cdot, \cdot)$ is the set of all $u \in H$ such that

(i) ${ }^{\circ} \mathcal{E}_{1}(u, u)={ }^{\circ}[\mathcal{E}(u, u)+\langle u, u\rangle]<\infty$.

(ii) For all $t \in T, t \approx 0, \mathcal{E}\left(Q^{t} u, Q^{t} u\right) \approx \mathcal{E}(u, u)$.

If $\delta \in T$, let $T_{\delta}$ be the subline

$$
T_{\delta}=\{0, \delta, 2 \delta, \ldots\} \text {. }
$$

We write $X^{(\delta)}$ for the restriction $X \mid T_{\delta}$. For each $t \in T_{\delta}$, let $\mathcal{F}_{t}^{(\delta)}$ be the internal algebra on $\Omega$ generated by the sets

$$
[\omega]_{t}^{(\delta)}=\left\{\omega^{\prime} \in \Omega \mid X^{(\delta)}\left(\omega^{\prime}, s\right)=X^{(\delta)}(\omega, s) \text { for all } s \in T_{\delta}, s \leqslant t\right\} .
$$

It is easy to verify the following for all $k \in \mathbb{N}$,

$$
P\left([\omega]_{k \delta}^{(\delta)}\right)=m(\omega(0)) \prod_{n=0}^{k-1} q_{\omega(n \delta), \omega((n+1) \delta)}^{(\delta)}
$$


and

$$
P_{i}\left([\omega]_{k \delta}^{(\delta)}\right)=\delta_{i \omega(0)} \prod_{n=0}^{k-1} q_{\omega(n \delta), \omega((n+1) \delta)}^{(\delta)} .
$$

Therefore, for any $t \in T_{\delta}$ and $u \in H$, we have

$$
E_{i} u\left(X^{(\delta)}(t)\right)=Q^{t} u(i)=E_{i} u(X(t)) .
$$

In particular, we get

$$
E_{i} u\left(X^{(\delta)}(\delta)\right)=Q^{\delta} u(i)=\sum_{j=1}^{N} q_{i j}^{(\delta)} u(j) .
$$

This implies that the semigroup of $X^{(\delta)}$ is $\left\{Q^{t} \mid t \in T_{\delta}\right\}$. The infinitesimal generator $A^{(\delta)}$ of $X^{(\delta)}$ is given by

$$
A^{(\delta)} u(i)=\frac{1}{\delta}\left(u(i)-\sum_{j=1}^{N} u(j) q_{i j}^{(\delta)}\right) .
$$

Moreover, we can easily show that

$$
\begin{aligned}
& \sum_{j=0}^{N} q_{i j}^{(\delta)}=1 \text { for all } i \in S, \\
& q_{0 i}^{(\delta)}=0 \text { for all } i \neq 0
\end{aligned}
$$

and

$$
m_{i} q_{i j}^{(\delta)}=m_{j} q_{j i}^{(\delta)} \text { for all } i \neq 0, j \neq 0 \text {. }
$$

The Dirichlet form associated with $Q^{(\delta)}$ and $m$ is defined to be

$$
\mathcal{E}^{(\delta)}(u, v)=\left\langle A^{(\delta)} u, v\right\rangle=\sum_{i=1}^{N} A^{(\delta)} u(i) v(i) m(i) .
$$

Therefore, we have

$$
\begin{aligned}
\mathcal{E}^{(\delta)}(u, v)= & \frac{1}{\delta} \sum_{i=1}^{N}\left[u(i) v(i) m(i)-\sum_{j=1}^{N} u(j) v(i) q_{i j}^{(\delta)} m(i)\right] \\
= & \frac{1}{\delta}\left[\sum_{1 \leqslant i<j \leqslant N}(u(i)-u(j))(v(i)-v(j)) q_{i j}^{(\delta)} m(i)\right. \\
& \left.+\sum_{i=1}^{N} u(i) v(i) q_{i 0}^{(\delta)} m(i)\right]
\end{aligned}
$$


Similarly, the domain $\mathcal{D}\left(\mathcal{E}^{(\delta)}\right)$ of $\mathcal{E}^{(\delta)}(\cdot, \cdot)$ is the set of all $u \in H$ such that

(i) ${ }^{\circ} \mathcal{E}_{1}^{(\delta)}(u, u)={ }^{\circ}\left[\mathcal{E}^{(\delta)}(u, u)+(u, u)\right]<\infty$.

(ii) For all $t \in T_{\delta}, t \approx 0, \mathcal{E}^{(\delta)}\left(Q^{t} u, Q^{t} u\right) \approx \mathcal{E}^{(\delta)}(u, u)$.

For $\delta \in T, \alpha \in{ }^{*} \mathbf{R}_{+}$, we will use the following notations:

$$
\mathcal{E}^{(\delta)}(u)=\mathcal{E}^{(\delta)}(u, u), \quad \mathcal{E}_{\alpha}^{(\delta)}(u)=\mathcal{E}^{(\delta)}(u, u)+\alpha\langle u, u\rangle .
$$

\section{Exceptionality and Capacity Theory}

\subsection{EXCEPTIONAL SETS}

For $\delta \in T$, denote

$$
T^{\text {fin }}=\{t \in T \mid t \text { is finite }\}, \quad T_{\delta}^{\text {fin }}=\left\{t \in T_{\delta} \mid t \text { is finite }\right\},
$$

and for $r \in T$, let

$$
T^{r}=\{t \in T \mid t \leqslant r\}, \quad T_{\delta}^{r}=\left\{t \in T_{\delta} \mid t \leqslant r\right\} .
$$

Moreover, we know that $X^{(\delta)}$ is the restriction $X \mid T_{\delta}$.

For every $y \in Y$, let us define the monad $\mu(y)$ of $y$ by

$$
\mu(y)=\bigcap\left\{{ }^{*} O \mid O \text { is open such that } y \in O\right\} .
$$

We call a point $y \in \in^{*} Y$ is nearstandard if and only if $y \in \mu(x)$ for some $x \in Y$. Denote by $N s\left({ }^{*} Y\right)$ the set of all nearstandard points in ${ }^{*} Y$. Since $Y$ is a Hausdorff topological space, each element $y \in N s\left({ }^{*} Y\right)$ is nearstandard to exactly one element $x$ in $Y$. We call $x$ the standard part of $y$ and denote it by ${ }^{\circ} y$ or $s t(y)$. In particular, we can take $Y=\mathbf{R}$ and use this notation also.

DEFINITION 3.1. (i) A subset $B$ of $S_{0}$ is called $\delta$-exceptional if

$$
L(P)\left\{\omega \mid \exists t \in T_{\delta}^{\mathrm{fin}}(X(\omega, t) \in B)\right\}=0 .
$$

(ii) A subset $B$ of $S_{0}$ is called exceptional if it is $\delta$-exceptional for some infinitesimal $\delta \in T$.

REMARK 3.1. [3] has defined the $\delta$-exceptional set in the following manner

$$
L(P)\left\{\omega \mid\left(X(\omega, 0) \in \bar{S}_{0}\right) \wedge\left(\exists t \in T_{\delta}^{\mathrm{fin}}(X(\omega, t) \in B)\right)\right\}=0,
$$

where $\bar{S}_{0}=S_{0} \cap N s\left({ }^{*} Y\right)$. Therefore, if a subset $B$ is $\delta$-exceptional in our sense of the Definition 3.1, it is $\delta$-exceptional in the sense of (3.5).

REMARK 3.2. From (3.4), we see for every exceptional set $B$

$$
L(P)\{\omega \mid X(\omega, 0) \in B\}=0 .
$$

This implies that $L(m)(B)=0$. 
LEMMA 3.1. (i) All internal subsets $B \subset S_{0}$ with $m(B) \approx 0$ are exceptional.

(ii) The families of exceptional and $\delta$-exceptional sets are closed under countable unions.

Proof. Easy!

DEFINITION 3.2. (i) A $\delta$-exceptional subset $A$ of $S_{0}$ is called properly $\delta$-exceptional if there is a family $\left\{B_{m, n} \mid m, n \in \mathbf{N}\right\}$ of internal subsets such that

$$
A=\bigcup_{m \in \mathbb{N}} \bigcap_{n \in \mathbb{N}} B_{m, n}
$$

and for all $s_{i} \notin A$,

$$
L\left(P_{i}\right)\left\{\omega \mid \exists t \in T_{\delta}^{\mathrm{fin}}(X(\omega, t) \in A)\right\}=0 .
$$

(ii) A subset $A$ of $S_{0}$ is called properly exceptional if it is properly $\delta$ exceptional for some $\delta \approx 0, \delta \in T$.

PROPOSITION 3.1. If $A \subset S_{0}$ is $\delta$-exceptional, there is a properly $\delta$-exceptional set $B \supset A$.

Proof. The proof of this result is similar to [3,5.4.7 Lemma]. For the reason of the conciseness, we omit the detail discussion.

\subsection{CAPACITY THEORY}

We now turn to study the capacity theory of the hyperfinite Dirichlet form $\mathcal{E}(\cdot, \cdot)$. Recall that for $\alpha \in{ }^{*} \mathbf{R}_{+}$, the form $\mathcal{E}_{\alpha}(\cdot, \cdot)$ is defined by

$$
\mathcal{E}_{\alpha}(u, v)=\mathcal{E}(u, v)+\alpha \int_{S_{0}} u v \mathrm{~d} m .
$$

Let $f: D_{f} \rightarrow{ }^{*} \mathbf{R}$ be an internal function defined on an internal subset $D_{f}$ of $S_{0}$, and let $\delta \in T$. Define a stopping time $\sigma_{f}^{(\delta)}$ by

$$
\sigma_{f}^{(\delta)}(\omega)=\min \left\{t \in T_{\delta} \mid X^{(\delta)}(\omega, t) \in D_{f}\right\}
$$

For $\alpha \in{ }^{*} \mathbf{R}, \alpha \geqslant 0$, set

$$
e_{\alpha}^{(\delta)}(f)(i)=E_{i}\left[(1+\alpha \delta)^{-\sigma_{f}^{(\delta)} / \delta} f\left(X^{(\delta)}\left(\sigma_{f}^{(\delta)}\right)\right)\right] .
$$

Denote

$$
\mathcal{L}(f)=\left\{g \mid g: S_{0} \rightarrow \mathbf{R} \text { is internal and }\left.g\right|_{D_{f}}=f\right\} .
$$

Then we have 
LEMMA 3.2. $\mathcal{E}_{\alpha}^{(\delta)}\left(e_{\alpha}^{(\delta)}(f), e_{\alpha}^{(\delta)}(f)\right)=\min \left\{\mathcal{E}_{\alpha}^{(\delta)}(g, g) \mid g \in \mathcal{L}(f)\right\}$.

Proof. Refer to [3, Chapter 5].

We shall call $e_{\alpha}^{(\delta)}(f)$ equilibrium $\alpha$-potential of $f$ associated with $\mathcal{E}^{(\delta)}(\cdot, \cdot)$. Denote

$$
\operatorname{Cap}_{\alpha}^{(\delta)}(f)=\mathcal{E}_{\alpha}^{(\delta)}\left(e_{\alpha}^{(\delta)}(f), e_{\alpha}^{(\delta)}(f)\right) .
$$

We call $\operatorname{Cap}_{\alpha}^{(\delta)}(f)$ the $\alpha$-capacity of $f$ associated with $\mathcal{E}^{(\delta)}(\cdot, \cdot)$. An internal function $f: D_{f} \rightarrow \mathbf{R}$ has finite energy of $\mathcal{E}^{(\delta)}(\cdot, \cdot)$ if

$$
{ }^{\circ} \mathcal{E}_{1}^{(\delta)}\left(e_{1}^{(\delta)}(f), e_{1}^{(\delta)}(f)\right)<\infty
$$

Whenever $\delta=\Delta t$, we abbreviate $e_{\alpha}^{(\delta)}(f)$ and $\operatorname{Cap}_{\alpha}^{(\delta)}(f)$ by $e_{\alpha}(f)$ and $\operatorname{Cap}_{\alpha}(f)$, respectively.

If $f$ constant one on its domain $A=D_{f}$, we write $e_{\alpha}^{(\delta)}(A)$ for $e_{\alpha}^{(\delta)}(f)$, $\operatorname{Cap}_{\alpha}^{(\delta)}(A)$ for $\operatorname{Cap}_{\alpha}^{(\delta)}(f)$. In this case, we shall call $e_{\alpha}^{(\delta)}(A)$ the equilibrium $\alpha$-potential of $A$ associated with $\mathcal{E}^{(\delta)}(\cdot, \cdot)$, and $\operatorname{Cap}_{\alpha}^{(\delta)}(A)$ the $\alpha$-capacity of $A$ associated with $\mathcal{E}^{(\delta)}(\cdot, \cdot)$.

LEMMA 3.3. Let $\delta \in T, \alpha \geqslant 0$ and $\alpha \in \mathbf{R}$, the following results hold:

(i) If $A$ and $B$ are two internal subsets of $S_{0}, A \subset B$, then

$$
\operatorname{Cap}_{\alpha}^{(\delta)}(A) \leqslant \operatorname{Cap}_{\alpha}^{(\delta)}(B) \text {. }
$$

(ii) If $A$ and $B$ are internal subsets of $S_{0}$, then we have

$$
\operatorname{Cap}_{\alpha}^{(\delta)}(A \cup B)+\operatorname{Cap}_{\alpha}^{(\delta)}(A \cap B) \leqslant \operatorname{Cap}_{\alpha}^{(\delta)}(A)+\operatorname{Cap}_{\alpha}^{(\delta)}(B) .
$$

Proof. (i) is clear. We show conclusion (ii).

(ii) Without loss of generality, let us assume that $\delta=\Delta t$. Since

$$
e_{\alpha}(A) \vee e_{\alpha}(B)=\frac{e_{\alpha}(A)+e_{\alpha}(B)}{2}+\frac{\left|e_{\alpha}(A)-e_{\alpha}(B)\right|}{2}
$$

and

$$
e_{\alpha}(A) \wedge e_{\alpha}(B)=\frac{e_{\alpha}(A)+e_{\alpha}(B)}{2}-\frac{\left|e_{\alpha}(A)-e_{\alpha}(B)\right|}{2},
$$

we have the following

$$
\begin{aligned}
\operatorname{Cap}_{\alpha} & (A \bigcup B)+\operatorname{Cap}_{\alpha}(A \bigcap B) \\
& \leqslant \mathcal{E}_{\alpha}\left(e_{\alpha}(A) \vee e_{\alpha}(B)\right)+\mathcal{E}_{\alpha}\left(e_{\alpha}(A) \wedge e_{\alpha}(B)\right) \\
& =\frac{1}{2} \varepsilon_{\alpha}\left(e_{\alpha}(A)+e_{\alpha}(B)\right)+\frac{1}{2} \mathcal{E}_{\alpha}\left(\left|e_{\alpha}(A)-e_{\alpha}(B)\right|\right) \\
& \leqslant \frac{1}{2} \mathcal{E}_{\alpha}\left(e_{\alpha}(A)+e_{\alpha}(B)\right)+\frac{1}{2} \mathcal{E}_{\alpha}\left(e_{\alpha}(A)-e_{\alpha}(B)\right) \\
& =\mathcal{E}_{\alpha}\left(e_{\alpha}(A), e_{\alpha}(A)\right)+\mathcal{E}_{\alpha}\left(e_{\alpha}(B), e_{\alpha}(B)\right) \\
& =\operatorname{Cap}_{\alpha}(A)+\operatorname{Cap}_{\alpha}(B) .
\end{aligned}
$$


Now let us generalize our capacity theory. For every subset $A$ of $S_{0}$, we define

$$
{ }^{\circ} \mathrm{Cap}_{\alpha}^{(\delta)}(A)=\inf \left\{{ }^{\circ} \operatorname{Cap}_{\alpha}^{(\delta)}(B) \mid A \subset B, B \in \mathcal{S}_{0}\right\} .
$$

We call $\operatorname{Cap}_{\alpha}^{(\delta)}(A)$ the $\alpha$-capacity of $A$ associated with $\mathcal{E}^{(\delta)}(\cdot, \cdot)$.

LEMMA 3.4. Let $\delta \in T, \alpha \geqslant 0$ and $\alpha \in{ }^{*} \mathbf{R}$, the following results hold:

(i) If $\left\{A_{n} \mid n \in \mathbf{N}\right\}$ is an increasing sequence of internal subsets of $S_{0}$, then

$$
{ }^{\circ} \operatorname{Cap}_{\alpha}^{(\delta)}\left(\bigcup_{n \in \mathbf{N}} A_{n}\right)=\sup \left\{{ }^{\circ} \operatorname{Cap}_{\alpha}^{(\delta)}\left(A_{n}\right) \mid n \in \mathbf{N}\right\} \text {. }
$$

(ii) If $\left\{A_{n} \mid n \in \mathbf{N}\right\}$ is a decreasing sequence of internal subsets of $S_{0}$, then

$$
{ }^{\circ} \operatorname{Cap}_{\alpha}^{(\delta)}\left(\bigcap_{n \in \mathbb{N}} A n\right)=\inf \left\{{ }^{\circ} \operatorname{Cap}_{\alpha}^{(\delta)}\left(A_{n}\right) \mid n \in \mathbf{N}\right\} \text {. }
$$

Proof. (i) Set $a=\sup \left\{{ }^{\circ} \operatorname{Cap}_{\alpha}^{(\delta)}\left(A_{n}\right) \mid n \in \mathbf{N}\right\}$. Obviously, we have

$$
a \leqslant{ }^{\circ} \operatorname{Cap}_{\alpha}^{(\delta)}\left(\bigcup_{n \in \mathbb{N}} A_{n}\right) .
$$

Hence we can suppose that $a<\infty$. Let $\left\{A_{n} \mid n \in \mathrm{N}\right\}$ be an increasing internal extension of $\left\{A_{n} \mid n \in \mathbf{N}\right\}$. Given $\varepsilon>0$, we consider the following internal set

$$
\left\{n \in{ }^{*} \mathrm{~N} \mid \bigcup_{l=1}^{n} A_{l}=A_{n} \text { is internal, } \operatorname{Cap}_{\alpha}^{(\delta)}\left(\bigcup_{l=1}^{n} A_{l}\right)=\operatorname{Cap}_{\alpha}^{(\delta)}\left(A_{n}\right) \leqslant a+\varepsilon\right\} .
$$

It is easy to see that $\mathbf{N}$ is contained in above internal set. By saturation, there is an infinite member $M$ belonging to it. Therefore, we have

$$
\operatorname{Cap}_{\alpha}^{(\delta)}\left(\bigcup_{n \in \mathbb{N}} A_{n}\right) \leqslant \operatorname{Cap}_{\alpha}^{(\delta)}\left(A_{M}\right) \leqslant a+\varepsilon
$$

By letting $\varepsilon \downarrow 0$, we get

$$
{ }^{\circ} \mathrm{Cap}_{\alpha}^{(\delta)}\left(\bigcup_{n \in \mathrm{N}} A_{n}\right) \leqslant a .
$$

Therefore, we have

$$
{ }^{\circ} \mathrm{Cap}_{\alpha}^{(\delta)}\left(\bigcup_{n \in \mathrm{N}} A_{n}\right)=\sup \left\{{ }^{\circ} \mathrm{Cap}_{\alpha}^{(\delta)}\left(A_{n}\right) \mid n \in \mathrm{N}\right\} \text {. }
$$

(ii) In a similar way as (i), we can prove (ii). 
LEMMA 3.5.If $\left\{A_{n} \mid n \in \mathbf{N}\right\}$ is a sequence of internal subsets of $S_{0}$, then we have

$$
{ }^{\circ} \mathrm{Cap}_{\alpha}^{(\delta)}\left(\bigcup_{n \in \mathrm{N}} A_{n}\right) \leqslant \sum_{n \in \mathbf{N}}{ }^{\circ} \mathrm{Cap}_{\alpha}^{(\delta)}\left(A_{n}\right)
$$

for all $\delta \in T, \alpha \geqslant 0$.

Proof. Denote $b=\Sigma_{n \in \mathbb{N}}{ }^{\circ} \operatorname{Cap}_{\alpha}^{(\delta)}\left(A_{n}\right)$. If $b=\infty$, the inequality (3.18) holds. In the following proof, we assume $b<\infty$. Let $\left\{A_{n} \mid n \in \mathbb{N}\right\}$ be an internal extension of $\left\{A_{n} \mid n \in \mathbf{N}\right\}$. For every $\varepsilon>0$, it follows from Lemma 3.3 (ii) that

$$
\operatorname{Cap}_{\alpha}^{(\delta)}\left(\bigcup_{l=1}^{n} A_{l}\right) \leqslant \sum_{l=1}^{n} \operatorname{Cap}_{\alpha}^{(\delta)}\left(A_{l}\right) \leqslant \varepsilon+b \text { for all } n \in \mathbf{N} .
$$

Consider the following internal set

$$
\left\{n \in \mathbb{N} \mid \bigcup_{l=1}^{n} A_{l} \text { is internal and } \operatorname{Cap}_{\alpha}^{(\delta)}\left(\bigcup_{l=1}^{n} A_{l}\right) \leqslant \sum_{l=1}^{n_{b}} \operatorname{Cap}_{\alpha}^{(\delta)}\left(A_{l}\right) \leqslant b+\varepsilon\right\} .
$$

By saturation, there is an infinite element $M=M(\varepsilon)$ belonging to the above internal set. Hence, we obtain

$$
\operatorname{Cap}_{\alpha}^{(\delta)}\left(\bigcup_{l \in \mathbb{N}} A_{l}\right) \leqslant \operatorname{Cap}_{\alpha}^{(\delta)}\left(\bigcup_{l=1}^{M} A_{l}\right) \leqslant \sum_{l=1}^{M} \operatorname{Cap}_{\alpha}^{(\delta)}\left(A_{l}\right) \leqslant b+\varepsilon
$$

By letting $\varepsilon \downarrow 0$, we have proved (3.18).

PROPOSITION 3.2. For all $\delta \in T, \alpha \geqslant 0$, we have

(i) If $A$ and $B$ are two subsets of $S_{0}, A \subset B$, then

$$
\operatorname{Cap}_{\alpha}^{(\delta)}(A) \leqslant \operatorname{Cap}_{\alpha}^{(\delta)}(B)
$$

(ii) Let $\left\{A_{n} \mid n \in \mathbf{N}\right\}$ be a sequence of subsets of $S_{0}$, then

$$
{ }^{\circ} \mathrm{Cap}_{\alpha}^{(\delta)}\left(\bigcup_{n \in \mathbb{N}} A_{n}\right) \leqslant \sum_{n \in \mathbb{N}}{ }^{\circ} \operatorname{Cap}_{\alpha}^{(\delta)}\left(A_{n}\right) \text {. }
$$

(iii) Let $\left\{A_{n} \mid n \in \mathbf{N}\right\}$ be an increasing sequence of subsets of $S_{0}$, then

$$
{ }^{\circ} \mathrm{Cap}_{\alpha}^{(\delta)}\left(\bigcup_{n \in \mathbb{N}} A_{n}\right)=\sup \left\{{ }^{\circ} \mathrm{Cap}_{\alpha}^{(\delta)}\left(A_{n}\right) \mid n \in \mathbf{N}\right\} \text {. }
$$


Proof. (i) Easy! Using (i) and Lemma 3.5, we may show (ii).

(iii) We may assume that for all $n \in \mathbf{N}$,

$$
{ }^{\circ} \mathrm{Cap}_{\alpha}^{(\delta)}\left(A_{n}\right)<\infty \text {. }
$$

Given $\varepsilon>0$, for each $n \in \mathbf{N}$, let $B_{n}$ be an internal subset of $S_{0}$ such that

$$
A_{n} \subset B_{n}, \quad \operatorname{Cap}_{\alpha}^{(\delta)}\left(B_{n}\right) \leqslant \operatorname{Cap}_{\alpha}^{(\delta)}\left(A_{n}\right)+\varepsilon .
$$

Then we have from (3.19) and Lemma 3.4 (i) that

$$
\begin{gathered}
{ }^{\circ} \operatorname{Cap}_{\alpha}^{(\delta)}\left(\bigcup_{n \in \mathbb{N}} A_{n}\right) \leqslant{ }^{\circ} \operatorname{Cap}_{\alpha}^{(\delta)}\left(\bigcup_{n \in \mathbb{N}} B_{n}\right)=\sup _{n \in \mathbb{N}}\left\{{ }^{\circ} \operatorname{Cap}_{\alpha}^{(\delta)}\left(B_{n}\right)\right\} \\
\leqslant \sup _{n \in \mathbb{N}}\left\{\left(\operatorname{Cap}_{\alpha}^{(\delta)}\left(A_{n}\right)+\varepsilon\right)\right\} \leqslant \sup _{n \in \mathbf{N}}\left\{{ }^{\circ} \operatorname{Cap}_{\alpha}^{(\delta)}\left(A_{n}\right)\right\}+\varepsilon .
\end{gathered}
$$

By letting $\varepsilon \downarrow 0$, we get

$$
{ }^{\circ} \mathrm{Cap}_{\alpha}^{(\delta)}\left(\bigcup_{n \in \mathrm{N}} A_{n}\right) \leqslant \sup \left\{{ }^{\circ} \operatorname{Cap}_{\alpha}^{(\delta)}\left(A_{n}\right) \mid n \in \mathbf{N}\right\} .
$$

On the other hand, it is easy to see

$$
{ }^{\circ} \operatorname{Cap}_{\alpha}^{(\delta)}\left(\bigcup_{n \in \mathbb{N}} A_{n}\right) \geqslant \sup \left\{{ }^{\circ} \operatorname{Cap}_{\alpha}^{(\delta)}\left(A_{n}\right) \mid n \in \mathbf{N}\right\} .
$$

From (3.22) and (3.23), we have proved Proposition 3.2 (iii).

For the purpose of explaining our Theorem 3.1 in the following, we first introduce some notations in capacity theory (refer to [10]). Let $G$ be a set, $\mathcal{G}$ be a family of some subsets of $G$. Denote by $\mathcal{G}_{\sigma}$ (respectively, $\mathcal{G}_{\delta}$ ) the closure of a collection of subsets of $G$ under countable union (respectively, countable intersection). That is,

$$
\mathcal{G}_{\sigma}=\left\{\bigcup_{n=1}^{\infty} A_{n} \mid A_{n} \in \mathcal{G}\right\}, \quad \mathcal{G}_{\delta}=\left\{\bigcap_{n=1}^{\infty} A_{n} \mid A_{n} \in \mathcal{G}\right\} .
$$

Moreover, we denote $\mathcal{G}_{\sigma \delta}=\left(\mathcal{G}_{\sigma}\right)_{\delta}$.

DEFINITION 3.3. Let $G$ be a set. A paving $\mathcal{G}$ on $G$ is a family of subsets of $G$ such that the empty set $\phi$ is contained in $\mathcal{G}$. The pair $(G, \mathcal{G})$ consisting of a set $G$ and a paving $\mathcal{G}$ on $G$ is called a paved set.

DEFINITION 3.4. Let $(G, \mathcal{G})$ be a paved set. The paving $\mathcal{G}$ is said to be semicompact if every countable family of elements of $\mathcal{G}$, which has the finite intersection property, has a nonempty intersection. 
It is easy to see that $\left(S_{0}, \mathcal{S}_{0}\right)$ is a semi-compact paved set. Moreover, $\mathcal{S}_{0}$ is closed under the complement, finite union and finite intersection operations.

DEFINITION 3.5. A subset $A$ of $S_{0}$ is said to be $S_{0}$-analytic if there exists an auxiliary set $G$ with a semi-compact paving $\mathcal{G}$, and a subset $B \subset G \times S_{0}$ belonging to $\left(\mathcal{G} \times \mathcal{S}_{0}\right)_{\sigma \delta}$ such that $A$ is the projection of $B$ on $S_{0}$. We denote by $\mathcal{A}\left(\mathcal{S}_{0}\right)$ all the $\mathcal{S}_{0}$-analytic sets (Notice that $\mathcal{G} \times \mathcal{S}_{0}=\left\{G_{1} \times S_{1} \mid G_{1} \in \mathcal{G}\right.$ and $\left.S_{1} \in \mathcal{S}_{0}\right\}$ ).

LEMMA 3.6. The $\sigma$-field $\sigma\left(\mathcal{S}_{0}\right)$ generated by $\mathcal{S}_{0}$ is contained in $\mathcal{A}\left(\mathcal{S}_{0}\right)$.

Proof. For every $F \in \mathcal{S}_{0}, S_{0}-F$ belongs to $\mathcal{S}_{0}$ also. By [10, Chapter III T12 Theorem], we know $\sigma\left(\mathcal{S}_{0}\right) \subset \mathcal{A}\left(\mathcal{S}_{0}\right)$.

DEFINITION 3.6. An extended real valued set function

$$
I: 2^{S_{0}} \rightarrow[-\infty,+\infty]
$$

defined on all subsets $2^{S_{10}}$ of $S_{0}$, is called a Choquet $\mathcal{S}_{0}$-capacity if it satisfies the following properties:

(i) $I$ is increasing, i.e.,

$$
A \subset B \Rightarrow I(A) \leqslant I(B) \text {. }
$$

(ii) For every inceasing sequence $\left\{A_{n} \mid n \in \mathbf{N}\right\}$ of subsets of $S_{0}$ :

$$
I\left(\bigcup_{n \in \mathbf{N}} A_{n}\right)=\sup _{n \in \mathbf{N}} I\left(A_{n}\right) .
$$

(iii) For every decreasing sequence $\left\{A_{n} \mid n \in \mathbf{N}\right\}$ of elements of $\mathcal{S}_{0}$ :

$$
I\left(\bigcap_{n \in \mathbf{N}} A_{n}\right)=\inf _{n \in \mathbf{N}} I\left(A_{n}\right) .
$$

We have reached one of our main results.

THEOREM 3.1. For each $\delta \in T$ and $\alpha \geqslant 0, \alpha \in \mathbf{R}^{*}$, we have the following results:

(i) ${ }^{\circ} \mathrm{Cap}_{\alpha}^{(\delta)}(\cdot)$ is a Choquet $\mathcal{S}_{0}$-capacity.

(ii) Every $\mathcal{S}_{0}$-analytic set is capacitable with respect to capacity ${ }^{\circ} \mathrm{Cap}_{\alpha}^{(\delta)}(\cdot)$. That is, for every $A \in \mathcal{A}\left(\mathcal{S}_{0}\right)$, we have

$$
\begin{aligned}
& { }^{\circ} \mathrm{Cap}_{\alpha}^{(\delta)}(A) \\
& \quad=\sup \left\{{ }^{\circ} \mathrm{Cap}_{\alpha}^{(\delta)}(B) \mid B=\bigcap_{m \in \mathbf{N}} B_{m}, B_{m} \in \mathcal{S}_{0} \text { and } B \subset A\right\} .
\end{aligned}
$$


(iii) Every subset $A$ of $S_{0}$ belonging to $\sigma\left(\mathcal{S}_{0}\right)$ is capacitable with respect to the capacity ${ }^{\circ} \mathrm{Cap}_{\alpha}^{(\delta)}(\cdot)$ whenever $0<\operatorname{st}(\alpha)<\infty$.

Proof. By Lemma 3.3 and Proposition 3.2, we know that ${ }^{\circ} \mathrm{Cap}_{\alpha}^{(\delta)}(\cdot)$ is a Choquet $\mathcal{S}_{0}$-capacity. Therefore, (ii) hold by [10, Chapter III T19 Theorem]. (iii) is the consequence of (ii) and Lemma 3.6.

DEFINITION 3.7. (i) A subset $B$ of $S_{0}$ is called $\delta$-zero capacity, if we have $\operatorname{Cap}_{1}^{\langle\delta\rangle}(B) \approx 0$.

(ii) A subset $B$ of $S_{0}$ is called zero capacity if $\operatorname{Cap}_{1}^{(\delta)}(B) \approx 0$ for some infinitesimal $\delta \in T$.

REMARK 3.3. For any $B \in \mathcal{S}_{0}$ and $\delta \in T$, we have

$$
m_{(}(B) \leqslant \operatorname{Cap}_{i}^{(\delta)}(B) .
$$

Therefore, for any zero capacity subset $B$ of $S_{0}$, we have

$$
L(m)(B)=0 .
$$

In regular Dirichlet space theory, we know the concepts of exceptional sets and zero capacity sets are equivalent, see [7, Theorem 4.3.1]. As the third part in this section, we will discuss the similar problem in our hyperfinite Dirichlet space theory.

\subsection{RELATIONSHIP BETWEEN FAMILY OF EXCEPTIONAL SETS AND FAMILY OF ZERO CAPACITY SETS}

LEMMA 3.7. Let $\left\{B_{n} \mid n \in \mathbf{N}\right\}$ be a sequence of internal subsets of $S_{0}$. If $\lim _{n \rightarrow \infty}{ }^{\circ} \operatorname{Cap}_{1}^{(\delta)}\left(\bigcap_{m=1}^{n} B_{m}\right)=0$, then $\bigcap_{n=1}^{\infty} B_{n}$ is a $\delta$-exceptional set, where $\delta \in T$.

Proof. Since $\mathcal{S}_{0}$ is closed under finite intersection, we may assume that $\left\{B_{n} \mid n \in \mathbf{N}\right\}$ is a decreasing sequence. Define a stopping time for each $n \in \mathbf{N}$,

$$
\sigma_{B_{n}}^{(\delta)}(\omega)=\min \left\{t \in T_{\delta} \mid X(\omega, t) \in B_{n}\right\} .
$$

Because

$$
\begin{aligned}
L(P) & \left\{\omega \mid \exists t \in T_{\delta}^{1}\left(X(\omega, t) \in B_{n}\right)\right\} \\
& ={ }^{\circ} P\left\{\omega \mid \exists t \in T_{\delta}^{1}\left(X(\omega, t) \in B_{n}\right)\right\} \\
& =\int_{S_{0}} E_{i}\left(\omega \mid \exists t \in T_{\delta}^{1}\left(X(\omega, t) \in B_{n}\right)\right) \mathrm{d} m(i) \\
& =\int_{S_{0}} E_{i} 1_{\left(\sigma_{\mathbf{B}_{n}}^{(\delta)} \leqslant 1\right)} \mathrm{d} m(i)
\end{aligned}
$$




$$
\begin{aligned}
& =\int_{S_{0}} E_{i}\left\{\omega \mid(1+\delta)^{-\sigma_{B_{n}}^{(\delta)} / \delta} \geqslant(1+\delta)^{-(1 / \delta)}\right\} \mathrm{d} m(i) \\
& \leqslant \int_{S_{0}} E_{i}\left\{\frac{(1+\delta)^{-\sigma_{B_{n}}^{(\delta)} / \delta}}{(1+\delta)^{-(1 / \delta)}}\right\} \mathrm{d} m(i) \\
& =e \cdot \int_{S_{0}} E_{i}(1+\delta)^{-\sigma_{B_{n}}^{(\delta)} / \delta} \mathrm{d} m(i)=e \cdot \int_{S_{0}} e_{1}^{(\delta)}\left(B_{n}\right)(i) \mathrm{d} m(i) \\
& \leqslant e \cdot{ }^{\circ} \mathcal{E}_{1}\left(e_{1}^{(\delta)}\left(B_{n}\right)_{2} e_{1}^{(\delta)}\left(B_{n}\right)\right)=e \cdot{ }^{\circ} \operatorname{Cap}_{1}^{(\delta)}\left(B_{n}\right) \rightarrow 0,
\end{aligned}
$$

where the last inequality comes from [3, Chapter 5, Section 5.3, Equation (35)]. From (3.31), we know the following

$$
L(P)\left\{\omega \mid \exists t \in T_{\delta}^{1}\left(X(\omega, t) \in \bigcap_{n=1}^{\infty} B_{n}\right)\right\}=0 .
$$

By the symmetric property of the Markov process $X(t)$ and (3.32), we have

$$
L(P)\left\{\omega \mid \exists t \in T_{\delta}^{\text {fin }}\left(X(\omega, t) \in \bigcap_{n=1}^{\infty} B_{n}\right)\right\}=0 .
$$

Therefore, the $\operatorname{set} \bigcap_{n=1}^{\infty} B_{n}$ is $\delta$-exceptional.

THEOREM 3.2.If a subset $A$ of $S_{0}$ is $\delta$-zero capacity, it is $\delta$-exceptional.

Proof. Since $\operatorname{Cap}_{1}^{(\delta)}(A) \approx 0$, we can take a sequence of internal subsets $\left\{B_{n} \mid n \in \mathbf{N}\right\}$ satisfying

$$
A \subset \bigcap_{n=1}^{\infty} B_{n}, \quad \lim _{n \rightarrow \infty}{ }^{\circ} \mathrm{Cap}_{1}^{(\delta)}\left(\bigcap_{m=1}^{n} B_{m}\right)=0 .
$$

Using Lemma 3.7, we know that $\bigcap_{n=1}^{\infty} B_{n}$ is $\delta$-exceptional. Hence $A$ is $\delta$-exceptional also. This completes the proof of Theorem 3.2.

LEMMA 3.8. Let $\delta_{1} \in T, \delta_{1} \approx 0$, and $A$ be a subset of $S_{0}$. If $A$ is $\delta_{1}$-exceptional and there exists an internal subset $B$ of $S_{0}$ such that

$$
A \subset B, \quad{ }^{\circ}\left[\operatorname{Cap}_{1}^{\left(\delta_{1}\right)}(B)\right]<\infty,
$$

then there is an infinitesimal $\delta_{0} \in T$ larger than $\delta_{1}$ such that $A$ is $\delta$-zero capacity for all $\delta \geqslant \delta_{0}$. 
Proof. By using Proposition 3.1, there exists a properly $\delta_{1}$-exceptional set

$$
\bigcup_{m \in \mathbb{N}} \bigcap_{n \in \mathbb{N}} B_{m, n} \supset A
$$

For simplicity, we assume that $B_{m, n} \subset B$ for all $n, m \in \mathbf{N}$, and for each $m$, the sequence $\left\{B_{m, n} \mid n \in \mathbf{N}\right\}$ is decreasing with respect to $n$. In order to show that $A$ is zero capacity, we first prove $\bigcap_{n \in N} B_{m, n}$ is zero capacity for every $m$. From now on, we fix an $m \in \mathbf{N}$.

By the assumption (3.34), we know that ${ }^{\circ} \mathrm{Cap}_{1}^{\left(\delta_{1}\right)}\left(B_{m, n}\right)<\infty$ for every $n$. Moreover, $\operatorname{Cap}_{1}^{\left(\delta_{1}\right)}\left(B_{m, n}\right)$ is decreasing with $n$. Therefore, we obtain

$$
\begin{aligned}
& { }^{\circ}\left[\mathcal{E}_{1}^{\left(\delta_{1}\right)}\left(e_{1}^{\left(\delta_{1}\right)}\left(B_{m, n}\right)-e_{1}^{\left(\delta_{1}\right)}\left(B_{m, k+n}\right)\right)\right] \\
& \quad={ }^{\circ}\left[\mathcal{E}_{1}^{\left(\delta_{1}\right)}\left(e_{1}^{\left(\delta_{1}\right)}\left(B_{m, n}\right)\right)-\mathcal{E}_{1}^{\left(\delta_{1}\right)}\left(e_{1}^{\left(\delta_{1}\right)}\left(B_{m, n+k}\right)\right)\right] \rightarrow 0 \text { as } n \rightarrow \infty .
\end{aligned}
$$

This means that $\left\{e_{1}^{\left(\delta_{1}\right)}\left(B_{m, n}\right) \mid n \in \mathbf{N}\right\}$ is a Cauchy sequence with respect to the inner product ${ }^{\circ} \mathcal{E}_{1}^{\left(\delta_{1}\right)}(\cdot, \cdot)$. Let $\left\{B_{m, n} \mid n \in \mathbb{N}\right\}$ be a decreasing extension of $\left\{B_{m, n} \mid n \in \mathbf{N}\right\}$. By saturation, there exists an infinite element $n_{0} \in \mathbf{N}-\mathbf{N}$ such that

$$
\lim _{n \rightarrow \infty}{ }^{\circ}\left[\mathcal{E}_{1}^{\left(\delta_{1}\right)}\left(e_{1}^{\left(\delta_{1}\right)}\left(B_{m, n}\right)-e_{1}^{\left(\delta_{1}\right)}\left(B_{m, n_{0}}\right)\right)\right]=0 .
$$

On the one hand, we have that

$$
{ }^{\circ}\left[\mathcal{E}_{1}^{\left(\delta_{1}\right)}\left(e_{1}^{\left(\delta_{1}\right)}\left(B_{m, n_{0}}\right), e_{1}^{\left(\delta_{1}\right)}\left(B_{m, n_{0}}\right)\right)\right]<\infty .
$$

By ([3], 5.1.9 Corollary on p. 232) we can find an infinitesimal $\delta_{m}=\delta_{1} k_{m}$ for some $k_{m} \in \mathbb{N}$ such that $e_{1}^{\left(\delta_{1}\right)}\left(B_{m, n_{0}}\right) \in \mathcal{D}\left(\mathcal{E}^{(\delta)}\right)$ for all $\delta \geqslant \delta_{m}, \delta \approx 0, \delta \in T_{\delta_{1}}$. On the other hand, for every $i \in S_{0}$, it is easy to see that $\left\{e_{1}^{\left(\delta_{1}\right)}\left(B_{m, n}\right)(i) \mid n \in \mathbf{N}\right\}$ is decreasing with respect to $n$. Denote

$$
e_{m}(i)=\lim _{n \rightarrow \infty}{ }^{\circ} e_{1}^{\left(\delta_{1}\right)}\left(B_{m, n}\right)(i)
$$

Since $\bigcup_{m \in \mathbb{N}} \bigcap_{n \in \mathbf{N}} B_{m, n}$ is properly $\delta_{1}$-exceptional, we know

$$
e_{m}(i)=0 \quad \text { for every } i \notin \bigcup_{m \in \mathbb{N}} \bigcap_{n \in \mathbb{N}} B_{m, n} .
$$

In fact, for every $M_{0} \in[0, \infty)$, we have

$$
\begin{aligned}
e_{1}^{(\delta)}\left(B_{m, n}\right)(i) & =E_{i}\left[\left(1+\delta_{1}\right)^{-\sigma_{m, n}^{\left(\delta_{1}\right)} / \delta_{1}}\right] \\
& =E_{i}\left[\left(1+\delta_{1}\right)^{-\sigma_{m, n}^{\left(\delta_{1}\right)} / \delta_{1}}\left(1_{\left(\sigma_{B_{m, n}} \geqslant M_{0}\right)}+1_{\left(\sigma_{m, n}<M_{0}\right)}\right)\right] \\
& \leqslant\left(1+\delta_{1}\right)^{-M_{0} / \delta_{1}}+E_{i} 1_{\left(\sigma_{m, n}<M_{0}\right)} .
\end{aligned}
$$


By letting $M_{0}$ sufficiently large, we know $\left(1+\delta_{1}\right)^{-M_{0} / \delta_{1}}$ will be very small. Taking $n$ sufficiently large, we see that (3.38) holds.

Since $e_{1}^{\left(\delta_{1}\right)}\left(B_{m, n_{0}}(i) \leqslant e_{1}^{\left(\delta_{1}\right)}\left(B_{m, n}\right)(i)\right.$ for every $n \in \mathbf{N}, i \in S_{0}$, we have from (3.38) that

$$
e_{1}^{\left(\delta_{1}\right)}\left(B_{m, n_{0}}\right)(i) \approx 0 \quad \text { for every } i \notin \bigcup_{m \in \mathrm{N}} \bigcap_{n \in \mathrm{N}} B_{m, n} .
$$

Now we have

$$
{ }^{\circ}\left(e_{1}^{\left(\delta_{1}\right)}\left(B_{m, n_{0}}\right)\right)=\operatorname{st}\left(e_{1}^{\left(\delta_{1}\right)}\left(B_{m, n_{0}}\right)\right)=0
$$

in $L^{2}\left(S_{0}, L\left(\mathcal{S}_{0}\right), L(m)\right)$ because

$$
L(m)\left(\bigcup_{m \in \mathbb{N}} \bigcap_{n \in \mathbb{N}} B_{m, n}\right)=0
$$

and $e_{1}^{\left(\delta_{1}\right)}\left(B_{m, n_{0}}\right) \in \mathcal{D}\left(\mathcal{E}^{(\delta)}\right)$ for all $\delta \geqslant \delta_{m}, \delta \approx 0, \delta \in T_{\delta_{1}}$, which implies $e_{1}^{\left(\delta_{1}\right)}\left(B_{m, n_{0}}\right)$ is $S^{2}$-integrable in the sense of ([3], p. 77, Chapter 3).

Therefore, we have

$$
\mathcal{E}_{1}^{(\delta)}\left(e_{1}^{\left(\delta_{1}\right)}\left(B_{m, n_{0}}\right), e_{1}^{\left(\delta_{1}\right)}\left(B_{m, n_{0}}\right)\right) \approx 0 \text { for all } \delta \geqslant \delta_{m}, \delta \approx 0, \delta \in T_{\delta_{1}} .
$$

Thus, we get

$$
{ }^{\circ}\left[\mathcal{E}_{1}^{(\delta)}\left(e_{1}^{(\delta)}\left(B_{m, n}\right), e_{1}^{(\delta)}\left(B_{m, n}\right)\right)\right] \rightarrow 0 \text { for all } \delta \geqslant \delta_{m}, \delta \approx 0, \delta \in T_{\delta_{1}} .
$$

This says that

$$
\operatorname{Cap}_{1}^{(\delta)}\left(\bigcap_{n \in \mathbf{N}} B_{m, n}\right) \approx 0 \text { for all } \delta \geqslant \delta_{m}, \delta \approx 0, \delta \in T_{\delta_{1}} .
$$

By saturation, there is a $\delta_{0} \approx 0, \delta_{0} \in T_{\delta_{1}}$ larger than all $\delta_{m}, m \in \mathbf{N}$. Therefore, it follows from (3.40) that for $\delta \geqslant \delta_{0}$

$$
\operatorname{Cap}_{i}^{(\delta)}\left(\bigcap_{n \in \mathbb{N}} B_{m, n}\right) \approx 0 \text { for all } m \in \mathbf{N} .
$$

By Proposition 3.2 (i) and (ii) and (3.41), we obtain

$$
{ }^{\circ} \operatorname{Cap}_{1}^{(\delta)}(A) \leqslant{ }^{\circ} \operatorname{Cap}_{1}^{\left(\delta_{0}\right)}\left(\bigcup_{m \in \mathbb{N}} \bigcap_{n \in \mathbb{N}} B_{m, n}\right) \leqslant \sum_{m=1}^{\infty}{ }^{\circ} \operatorname{Cap}_{1}^{\left(\delta_{0}\right)}\left(\bigcap_{n \in \mathbb{N}} B_{m, n}\right)=0 .
$$

Therefore, the set $A$ has $\delta$-zero capacity. 
THEOREM 3.3. For $\delta_{1} \in T, \delta_{1} \approx 0$, let $A$ be a subset of $S_{0}$. If $A$ is $\delta_{1}$-exceptional and there exists a sequence of internal subsets $\left\{B_{n} \mid n \in \mathbf{N}\right\}$ of $S_{0}$ such that

$$
A \subset \bigcup_{n \in \mathbf{N}} B_{n} \text { and }{ }^{\circ} \operatorname{Cap}_{1}^{\left(\delta_{1}\right)}\left(B_{n}\right)<\infty \quad \forall n \in \mathbf{N}
$$

then there is an infinitesimal $\delta_{0} \in T$ larger than $\delta_{1}$ such that $A$ is $\delta$-zero capacity for all $\delta \geqslant \delta_{0}$.

Proof. It is followed from Lemma 3.8 and Proposition 3.2 (ii).

\section{Measures of Hyperfinite Energy Integrals}

We know that for $\alpha \in \mathbf{R}^{*} \mathbf{R}, \alpha \geqslant 0$ and $\delta \in T$,

$$
\mathcal{E}_{\alpha}^{(\delta)}(u, v)=\mathcal{E}^{(\delta)}(u, v)+\alpha\langle u, v\rangle .
$$

Each of these forms generates a norm (possibly a seminorm in the case $\alpha=0$ ):

$$
|u|_{\alpha}^{(\delta)}=\left[\mathcal{E}_{\alpha}^{(\delta)}(u, u)\right]^{1 / 2} .
$$

Denote by $\operatorname{Fin}_{\alpha}^{(\delta)}(H)$ the set of all elements in $H$ with finite norm $|\cdot|_{\alpha}^{(\delta)}$. By defining $u \approx_{\alpha}^{(\delta)} v$ if $|u-v|_{\alpha}^{(\delta)} \approx 0$, the space

$$
{ }^{\circ} H_{\alpha}^{(\delta)}=\operatorname{Fin}_{\alpha}^{(\delta)}(H) / \approx_{\alpha}^{(\delta)}
$$

is a Hilbert space if ${ }^{\circ} \alpha>0$ with respect to the inner product

$$
\left.\left([u]_{\alpha}^{(\delta)},[v]_{\alpha}^{(\delta)}\right)_{\alpha}^{(\delta)}={ }^{o} \mathcal{E}_{\alpha}^{(\delta)}(u, v)\right],
$$

where $[u]_{\alpha}^{(\delta)}$ denotes the equivalence class of $u$ under the norm $|\cdot|_{\alpha}^{(\delta)}$, and $(\cdot, \cdot)_{\alpha}^{(\delta)}$ denotes the related inner product.

DEFINITION 4.1. Let $\mu$ be a hyperfinite positive measure on $S_{0}$. For $\delta \in T$, if there exists a constant $C \in *[0, \infty)=\mathbf{R}_{+}$such that

$$
\int_{S_{0}}|u(s)| \mu(\mathrm{d} s)=\sum_{i=1}^{N}\left|u\left(s_{i}\right)\right| \mu(i) \leqslant C\left[\mathcal{E}_{1}^{(\delta)}(u, u)\right]^{1 / 2}
$$

for every $u \in H$, we say that $\mu$ is of $\delta$-hyperfinite energy integral. Moreover, if there exists $C \in \mathbf{R}_{+}$satisfying (4.5) and ${ }^{\circ} C<\infty, \mu$ is said to be of $\delta$-finite energy integral.

Afterwards, we will identify a hyperfinite measure $\mu$ on $S_{0}$ with the measure $\tilde{\mu}$ on $S$ defined by $\tilde{\mu}\left(s_{0}\right)=0, \tilde{\mu}\left(s_{i}\right)=\mu\left(s_{i}\right)$ for all $s_{i} \in S_{0}$. 
THEOREM 4.1. A positive hyperfinite measure $\mu$ on $S_{0}$ is of $\delta$-hyperfinite energy integrals iff for each $\alpha \in{ }^{*} \mathbf{R}, 0<\operatorname{st}(\alpha)<\infty$, there exists an element $u \in H$ such that for every $v \in H$,

$$
\mathcal{E}_{\alpha}^{(\delta)}(v, u)=\int_{S_{0}} v(s) \mu(\mathrm{d} s)
$$

Moreover, if $\mu$ is of $\delta$-finite energy integral, we have $u \in \operatorname{Fin}_{\alpha}^{(\delta)}(H)$.

Proof. Follows from the Riesz's representation theorem.

REMARK 4.1. We call $u$ in Theorem $4.1 \alpha$-potential of $\mu$ associated with $\mathcal{E}^{(\delta)}(\cdot, \cdot)$, and denote it by $U_{\alpha}^{(\delta)} \mu$.

DEFINITION 4.2. Fix $\alpha \in{ }^{*} \mathbf{R}, \alpha \geqslant 0$ and $\delta \in T$. An element $u \in H$ is called hyperfinite $\alpha$-excessive associated with $\mathcal{E}^{(\delta)}(\cdot, \cdot)$, if

$$
u(i) \geqslant 0, Q^{\delta} u(i) \leqslant(1+\alpha \delta) u(i)
$$

for every $i \in S_{0}$ such that $m(i) \neq 0$.

In order to develop our theory, let $\left\{G_{\beta}^{(\delta)} \mid-\frac{1}{\delta}<\beta<0\right\}$ be the resolvent of $\mathcal{E}^{(\delta)}(\cdot, \cdot)$, i.e., it is defined by

$$
G_{\beta}^{(\delta)}=\left(A^{(\delta)}-\beta\right)^{-1}=\sum_{k=0}^{\infty}\left(Q^{\delta}+\beta \delta\right)^{k} \delta .
$$

Hence, we have for $\alpha E^{*} \mathbf{R}, 0<\alpha<1 / \delta$,

$$
\left(1+\alpha \delta-Q^{\delta}\right) G_{-\alpha}^{(\delta)}=\delta\left(\alpha+A^{(\delta)}\right) G_{-\alpha}^{(\delta)}=\delta .
$$

THEOREM 4.2.For $\delta \in T$, and $\alpha \in \mathbf{R}, \alpha \geqslant 0$, and $u \in H$. The following conditions are equivalent:

(1) $u$ is hyperfinite $\alpha$-excessive associated with $\mathcal{E}^{(\delta)}(\cdot, \cdot)$.

(2) There exists a hyperfinite positive measure is on $S_{0}$ such that

$$
\mathcal{E}_{\alpha}^{(\delta)}(u, v)=\int_{S_{0}} v(s) \mu(\mathrm{d} s) \text { for all } v \in H .
$$

(3) $\mathcal{E}_{\alpha}^{(\delta)}(u, v) \geqslant 0$ for all $v \in H, v \geqslant 0$.

(4) $u$ is an $\alpha$-potential of $\mathcal{E}^{(\delta)}(\cdot, \cdot)$. 
Proof. (1) $\Rightarrow(2)$. Assume that $u$ is hyperfinite $\alpha$-excessive associated with $\mathcal{E}^{(\delta)}(\cdot, \cdot)$. Define a hyperfinite positive measure $\mu$ on $S_{0}$ by

$$
\mu\left(s_{0}\right)=0, \mu\left(s_{i}\right)=\frac{1}{\delta}\left((1+\alpha \delta) u(i)-Q^{\delta} u(i)\right) m(i) \quad \text { for } \quad i \in S_{0} .
$$

Since

$$
\begin{aligned}
\mu\left(s_{i}\right) & =\frac{1}{\delta}\left((1+\alpha \delta) u(i)-Q^{\delta} u(i)\right) m(i) \\
& =\left(A^{(\delta)} u(i)+\alpha u(i)\right) m(i),
\end{aligned}
$$

we see that for every $v \in H$,

$$
\mathcal{E}_{\alpha}^{(\delta)}(u, v)=\sum_{i=1}^{N}\left(A^{(\delta)} u(i)+\alpha u(i)\right) v(i) m(i)=\int_{S_{0}} v(s) \mu(\mathrm{d} s)
$$

(2) $\Rightarrow$ (3). Easy!

(3) $\Rightarrow$ (1). Since

$$
\begin{aligned}
& \mathcal{E}_{\alpha}^{(\delta)}(|u|-u,|u|-u) \\
& \quad=\mathcal{E}_{\alpha}^{(\delta)}(|u|,|u|)-2 \mathcal{E}_{\alpha}^{(\delta)}(|u|-u, u)-\mathcal{E}_{\alpha}^{(\delta)}(u, u) \\
& \quad \leqslant-2 \mathcal{E}_{\alpha}^{(\delta)}(u,|u|-u) \leqslant 0,
\end{aligned}
$$

we obtain

$$
\mathcal{E}_{\alpha}^{(\delta)}(|u|-u,|u|-u)=0 .
$$

This implies that $u(i) \geqslant 0$ for every $i \in S$ such that $m(i) \neq 0$.

Furthermore, it follows from (4.9) that for any $v \in H$,

$$
\begin{aligned}
& \left\langle\left(1+\alpha \delta-Q^{\delta}\right) u, v\right\rangle=\left\langle u,\left(1+\alpha \delta-Q^{\delta}\right) v\right\rangle \\
& \quad=\mathcal{E}_{\alpha}^{(\delta)}\left(u,\left(1+\alpha \delta-Q^{\delta}\right) G_{-\alpha}^{(\delta)} v\right)=\mathcal{E}_{\alpha}^{(\delta)}(u, \delta v) .
\end{aligned}
$$

Fix $i \in S_{0}$. Let $v \in H$ be an internal function defined by $v(l)=\delta_{i l}, l \in S$. Then we have from (4.11) that

$$
\left(1+\alpha \delta-Q^{\delta}\right) u(i) m(i)=\mathcal{E}_{\alpha}^{(\delta)}(u, \delta v) \geqslant 0 .
$$

Therefore, the internal function $u$ is hyperfinite $\alpha$-excessive associated with $\mathcal{E}^{(\delta)}(\cdot, \cdot)$.

(2) $\Leftrightarrow$ (4). Follows from Theorem 4.1.

We denote by $\tau_{0}(\delta)$ the family of all internal positive measures on $S_{0}$ of $\delta$-hyperfinite energy integrals. Set $\tau_{0}=U\left\{\tau_{0}(\delta) \mid \delta\right.$ is infinitesimal, $\left.\delta \in T\right\}$. 
PROPOSITION 4.1. For $\delta \in T$, a hyperfinite positive measure $\mu$ on $S_{0}$ is of $\delta$-hyperfinite energy integral iff for any $\alpha \in \mathbf{R}, 0<\alpha<\frac{1}{\delta}$, there exists an $\alpha$-excessive function $u$ associated with $\mathcal{E}^{(\delta)}(\cdot, \cdot)$ such that

$$
\mu(i)=\frac{1}{\delta}\left((1+\alpha \delta) u(i)-Q^{\delta} u(i)\right) m(i) \quad \text { for all } i \in S_{0}
$$

Moreover, if $\bar{u} \in H$ satisfies above equation also, then $u(i)=\bar{u}(i)$ for all $i \in S_{0}$ with $m(i) \neq 0$.

REMARK 4.2. Let $u$ satisfy (4.12), then we have

$$
\mathcal{E}_{\alpha}^{(\delta)}(u, v)=\int_{S_{0}} v(s) \mu(\mathrm{d} s) \text { for all } v \in H .
$$

Proof of Proposition 4.1. $\Leftarrow$ Easy to see from Remark 4.2.

$\Rightarrow$ Assume that $\mu$ is of $\delta$-hyperfinite energy integral, let $u=U_{1}^{(\delta)} \mu \in H$ satisfy (4.6). Then we have

$$
\mu(i)=\left(G_{-1}^{(\delta)}\right)^{-1} u(i) m(i)=\frac{1}{\delta}\left((1+\delta) u(i)-Q^{\delta} u(i)\right) m(i) .
$$

Therefore, for any $\alpha \in{ }^{*} \mathbf{R}, 0<\alpha<\frac{1}{\delta}, v \in H$, we have

$$
\begin{aligned}
\int_{S} v(s) \mathrm{d} \mu(s) & =\int_{S} v(i)\left(G_{-1}^{(\delta)}\right)^{-1} u(i) \mathrm{d} m(i) \\
& =\mathcal{E}_{\alpha}^{(\delta)}\left(G_{-\alpha}^{(\delta)}\left(\left(G_{-1}^{(\delta)}\right)^{-1} u\right), v\right) .
\end{aligned}
$$

Hence by Theorem 4.2, $w=G_{-\alpha}^{(\delta)}\left(\left(G_{-1}^{(\delta)}\right)^{-1} u\right)$ is $\alpha$-excessive associated with $\mathcal{E}^{(\delta)}(\cdot, \cdot)$. Furthermore, we have

$$
\mu(i)=\frac{1}{\delta}\left((1+\alpha \delta) w(i)-Q^{\delta} w(i)\right) m(i) \quad \text { for all } \quad i \in S_{0} .
$$

PROPOSITION 4.2. For $\delta \in T$, and $\alpha \in{ }^{*} \mathbf{R}, \alpha \geqslant 0$. Let $u \in H$ be a hyperfinite $\alpha$-excessive function associated with $\mathcal{E}^{(\delta)}(\cdot, \cdot)$. Then for every $i \in S_{0}, m(i) \neq 0$. we have

$$
u(i) \geqslant 0,\left(u+\beta G_{\beta-\alpha}^{(\delta)} u\right)(i) \geqslant 0 \text { for all } \beta \in\left(\alpha-\frac{1}{\delta}, 0\right) .
$$

Proof. For $\beta \in\left(\alpha-\frac{1}{\delta}, 0\right)$, we have from (4.7) and (4.8) that

$$
\begin{aligned}
\beta G_{\beta-\alpha}^{(\delta)} u(i) & =\beta \sum_{k=0}^{\infty}\left(\left(Q^{\delta}+(-\alpha+\beta) \delta\right)^{k} \delta\right) u(i) \\
& \geqslant \beta \delta u(i) \sum_{k=0}^{\infty}(1+\beta \delta)^{k}=-u(i)
\end{aligned}
$$


Hence

$$
u(i)+\beta G_{\beta-\alpha}^{(\delta)} u(i) \geqslant 0
$$

PROPOSITION 4.3. For $\delta \in T$, let $\nu$ be a hyperfinite positive measure on $S_{0}$. Define a measure $\mu$ on $S_{0}$ by

$$
\mu(s)=\nu(s) 1_{(m(s) \neq 0)} \text { for } s \in S_{0} .
$$

Then $\mu$ is of $\delta$-hyperfinite energy integral.

Proof. Define

$$
f(s)=\frac{\nu(s)}{m(s)} 1_{(m(s) \neq 0)} \quad \text { for } \quad s \in S_{0}, f\left(s_{0}\right)=0 .
$$

For any $u \in H$, we have

$$
\begin{gathered}
\int_{S} u(s) \mathrm{d} \mu(s)=\int_{S} u(s) 1_{(m(s) \neq 0)} \mathrm{d} \nu(s) \\
=\int_{S} u(s) f(s) \mathrm{d} m(s)=\mathcal{E}_{1}^{(\delta)}\left(G_{-1}^{(\delta)} f, u\right) .
\end{gathered}
$$

Hence, $\mu$ is of $\delta$-hyperfinite energy integral.

THEOREM 4.3. For $\alpha \in{ }^{*} \mathbf{R}, 0<\operatorname{st}(\alpha)<\infty$ and $\delta \in T$. Let $\mu$ be a hyperfinite positive measure of $\delta$-finite energy integral, and $u$ be an $\alpha$-potential of $\mu$ associated with $\mathcal{E}^{(\delta)}(\cdot, \cdot)$. Define

$$
g_{n}(i)=n\left(u(i)-n G_{-\pi-\alpha}^{(\delta)} u(i)\right), \quad n=1,2, \ldots
$$

Then for every $v \in \operatorname{Fin}_{\alpha}^{(\delta)}(H)$, we have

(i) ${ }^{\circ} \mathcal{E}_{\alpha}^{(\delta)}\left(G_{-\alpha}^{(\delta)} g_{n}, v\right) \rightarrow E_{\alpha}^{(\delta)}\left({ }^{\circ} u,{ }^{\circ} v\right)$ as $n \rightarrow \infty$.

(ii) If $u \in \mathcal{D}\left(\mathcal{E}^{(\delta)}\right)$, let $\tilde{v} \approx_{\alpha}^{\delta} v$ and $\tilde{v} \in \mathcal{D}\left(\mathcal{E}^{(\delta)}\right)$,

$$
\lim _{n \rightarrow \infty} \int_{S_{0}}^{o} g_{n}(i) v(i) \mathrm{d} m(i)=\int_{S_{0}} \tilde{v}(i) \mathrm{d} \mu(i)
$$

Proof. (i) First of all, we have

$$
\begin{aligned}
{ }^{\circ} \mathcal{E}_{\alpha}^{(\delta)}\left(G_{-\alpha}^{(\delta)} g_{n}, v\right)= & { }^{\circ}\left(\left\langle g_{n}, v\right\rangle\right)={ }^{\circ}\left(\left\langle n u-n^{2} G_{-n-\alpha}^{(\delta)} u, v\right\rangle\right) \\
= & { }^{\circ}\left(\left\langle(n+\alpha) u-(n+\alpha)^{2} G_{-n-\alpha}^{(\delta)} u, v\right\rangle\right) \\
& +{ }^{\circ}\left(\left\langle-\alpha u+\alpha^{2} G_{-n-\alpha}^{(\delta)} u+2 n \alpha G_{-n-\alpha}^{(\delta)} u, v\right\rangle\right) .
\end{aligned}
$$


It follows from ([3], p. 238, 5.1.19 Theorem) that

$$
\begin{aligned}
& { }^{\circ}\left[\left((n+\alpha) u-(n+\alpha)^{2} G_{-n-\alpha}^{(\delta)} u, v\right\rangle+\alpha\langle u, v\rangle\right] \\
& \quad \rightarrow E_{\alpha}^{(\delta)}\left({ }^{\circ} u, v\right) \text { as } n \rightarrow \infty
\end{aligned}
$$

Moreover, let $\left\{e_{k} \mid 1 \leqslant k \leqslant N\right\}$ be an orthonormal basis of eigenvectors for $A^{(\delta)}$, and let $a_{k}$ be the $k$ th eigenvalue. Notice that if $u=\sum_{k=1}^{N} u_{k} e_{k}, v=\sum_{k=1}^{N} v_{k} e_{k}$, we have

$$
G_{-n-\alpha}^{(\delta)} u=\left(A^{(\delta)}+n+\alpha\right)^{-1} u=\sum_{k=1}^{N} \frac{u_{k}}{a_{k}+\bar{n}+\alpha} e_{k} .
$$

This implies that

$$
\left\langle-2 \alpha u+\left(\alpha^{2}+2 n \alpha\right) G_{-n-\alpha}^{(\delta)} u, v\right\rangle=-\alpha \sum_{k=1}^{N} \frac{2 a_{k}+\alpha}{a_{k}+n+\alpha} u_{k} v_{k}
$$

Therefore, we get

$$
\begin{aligned}
& { }^{\circ}\left|\left\langle-2 \alpha u+\left(\alpha^{2}+2 n \alpha\right) G_{-n-\alpha}^{(\delta)} u, v\right\rangle\right| \\
& \quad \leqslant\left[\frac{\alpha}{n}\left(\left|2 \mathcal{E}^{(\delta)}(u, v)\right|+|\alpha(u, v\rangle|\right)\right] \\
& \quad \rightarrow 0 \text { as } n \rightarrow \infty .
\end{aligned}
$$

From (4.17), (4.18) and (4.19), we know that (4.15) holds.

(ii) From the proof of (i) we get

$$
\lim _{n \rightarrow \infty} \int_{S_{0}}^{o} g_{n}(s) v(s) m(\mathrm{~d} s)={ }^{\circ} \mathcal{E}_{\alpha}^{(\delta)}(u, \tilde{v})=\int_{S_{0}} \tilde{v}(s) \mu(\mathrm{d} s)
$$

PROPOSITION 4.4. For $\delta \in T$, let $\mu$ be a positive measure of $\delta$-hyperfinite energy integral, then for every $L(\mu)$ measurable subset $A$ of $S_{0}$, we have

$$
L(\mu)(A) \leqslant\left[\sqrt{\mathcal{E}_{1}^{(\delta)}\left(U_{1}^{(\delta)} \mu, U_{1}^{(\delta)} \mu\right)} \sqrt{{ }^{\circ} \operatorname{Cap}_{1}^{(\delta)}(A)}\right] .
$$

Proof. For simplicity, we assume that ${ }^{\circ} \mathrm{Capl}^{(\delta)}(A)<\infty$.

If $A$ is internal, we have 


$$
\begin{aligned}
{ }^{\circ} \mu(A) & =\int_{S_{0}} 1_{A}(s) \mu(\mathrm{d} s) \leqslant \int_{S_{0}} e_{1}^{(\delta)}(A)(s) \mu(\mathrm{d} s) \\
& ={ }^{\circ} \mathcal{E}_{1}^{(\delta)}\left(e_{1}^{(\delta)}(A), U_{1}^{(\delta)} \mu\right) \\
& \leqslant\left[\sqrt{\mathcal{E}_{1}^{(\delta)}\left(U_{1}^{(\delta)} \mu, U_{1}^{(\delta)} \mu\right)} \sqrt{\mathcal{E}_{1}^{(\delta)}\left(e_{1}^{(\delta)}(A), e_{1}^{(\delta)}(A)\right)}\right] \\
& =\left[\sqrt{\mathcal{E}_{1}^{(\delta)}\left(U_{1}^{(\delta)} \mu, U_{1}^{(\delta)} \mu\right)} \sqrt{\operatorname{Cap}_{1}^{(\delta)}(A)}\right] .
\end{aligned}
$$

Now it is easy to see (4.20) holds for all $L(\mu)$ measurable subset $A$.

COROLLARY 4.1. Let $\mu$ be a positive measure of $\delta$-finite energy integral on $S_{0}$, then $L(\mu)$ charges no set of $\delta$-zero capacity.

Proof. It is clear from Proposition 4.4.

On the other hand, we have the following characterization theorem.

THEOREM 4.4. Let $A \subset S_{0}$ be an $\mathcal{A}\left(\mathcal{S}_{0}\right)$-measurable set (in particular, $A \in$ $\left.\sigma\left(\mathcal{S}_{0}\right)\right)$ and $\delta \in T$. We have

(1) For any $\mu \in \tau_{0}(\delta), L(\mu)(A)=0 \Longrightarrow \operatorname{Cap}_{1}^{(\delta)}(A)=0$.

(2) For any $\mu \in \tau_{00}(\delta), L(\mu)(A)=0 \Longrightarrow{ }^{\circ} \mathrm{Cap}_{1}^{(\delta)}(A)=0$,

where $\tau_{00}(\delta)=\left\{\mu \in \tau_{0}(\delta) \mid \mu\left(S_{0}\right)=1,{ }^{\circ}\left\|U_{1}^{(\delta)} \mu\right\|_{\infty}<\infty\right\} \quad$ and $\left\|U_{1}^{(\delta)} \mu\right\|_{\infty}=\max \left\{\left|U_{1}^{(\delta)} \mu(s)\right| \mid s \in S_{0}\right\}$.

Proof. (1) Assume that $\infty \geqslant{ }^{\circ} \mathrm{Capi}_{i}^{[\delta]}(A)=\alpha>0$. Since $A \in \mathcal{A}\left(\mathcal{S}_{0}\right)$, it follows from Theorem 3.1 (ii) that $A$ is capacitable with respect to the capacity ${ }^{\circ} \mathrm{Cap}_{1}^{(\delta)}(\cdot)$. That is

$$
{ }^{\circ} \mathrm{Cap}_{1}^{(\delta)}(A)=\sup \left\{{ }^{\circ} \mathrm{Cap}_{1}^{(\delta)}(B) \mid B=\bigcap_{n \in \mathbf{N}} B_{n}, B_{n} \in \mathcal{S}_{0} \quad \text { and } \quad B \subset A\right\} .
$$

Therefore, there exists a sequence $\left\{B_{n} \mid n \in \mathbf{N}\right\}$ of decreasing internal subsets of $S_{0}$ such that

$$
\bigcap_{n=1}^{\infty} B_{n} \subset A, \infty \geqslant \operatorname{Cap}_{1}^{(\delta)}\left(\bigcap_{n=1}^{\infty} B_{n}\right) \geqslant \frac{\alpha}{2}>0 .
$$


Let $\left\{B_{n} \mid n \in{ }^{*} \mathbf{N}\right\}$ be an internal decreasing extension of $\left\{B_{n} \mid n \in \mathbf{N}\right\}$. Then there exists an infinite $\gamma \in{ }^{*} \mathbf{N}-\mathbf{N}$ such that

$$
B_{\gamma} \subset A, 0<\frac{\alpha}{2} \leqslant{ }^{\circ} \operatorname{Cap}_{1}^{(\delta)}\left(\bigcap_{n=1}^{\infty} B_{n}\right)={ }^{\circ} \operatorname{Cap}_{1}^{(\delta)}\left(B_{\gamma}\right) \leqslant \infty .
$$

Set $B=B_{\gamma}$. Consider the internal function $e_{1}^{(\delta)}(B)$. Notice that if $i \notin B$, then

$$
\begin{gathered}
(1+\delta) e_{1}^{(\delta)}(B)(i)=(1+\delta) E_{i}\left[(1+\delta)^{-\sigma_{B}^{(\delta)} / \delta}\right] \\
=\sum_{j=1}^{N} e_{1}^{(\delta)}(B)(j) q_{i j}^{(\delta)}=Q^{\delta} e_{1}^{(\delta)}(B)(i)
\end{gathered}
$$

where $\sigma_{B}^{(\delta)}=\min \left\{t \in T_{\delta} \mid X(\omega, t) \in B\right\}$. If $i \in B$, then

$$
(1+\delta) e_{1}^{(\delta)}(B)(i)=(1+\delta) \geqslant \sum_{j=1}^{N} e_{1}^{(\delta)}(B)(j) q_{i j}^{(\delta)}=Q^{\delta} e_{1}^{(\delta)}(B)(i)
$$

It follows from (4.22) and (4.23) that the function $e_{1}^{(\delta)}(B)$ is hyperfinite 1-excessive associated with $\mathcal{E}^{(\delta)}(\cdot, \cdot)$. Define a hyperfinite positive measure $\mu$ on $S$ by

$$
\mu\left(s_{0}\right)=0, \quad \mu\left(s_{i}\right)=\frac{1}{\delta}\left((1+\delta) e_{1}^{(\delta)}(B)(i)-Q^{\delta} \mathrm{e}_{1}^{(\delta)}(B)(i)\right) m(i), \quad i \in S_{0} .
$$

Then we have

$$
\mu(B)=\int_{S_{0}} \mu(\mathrm{d} s)=\mathcal{E}_{1}^{(\delta)}\left(1, e_{1}^{(\delta)}(B)\right)=\operatorname{Cap}_{1}^{(\delta)}(B) .
$$

This contradicts to the assumption ${ }^{\circ} L(\mu)(A)=0$. Hence ${ }^{\circ} \mathrm{Cap}_{1}^{(\delta)}(A)=0$.

(2) Assume that $0<{ }^{\circ} \mathrm{Cap}_{1}^{(\delta)}(A)=\alpha \leqslant \infty$. By the proof $(1)$, we see that there exists an element $\nu \in \tau_{0}(\delta)$ such that (replace $\mu$ in the proof of (1) by $\nu$ )

$$
\begin{aligned}
& 0<{ }^{\circ} \operatorname{Cap}_{1}^{(\delta)}(B) \leqslant \infty, \quad \operatorname{Cap}_{1}^{(\delta)}(B)=\nu(B)=\nu\left(S_{0}\right) \\
& \text { and } \quad U_{1}^{(\delta)} \nu(i)=e_{1}^{(\delta)}(B)(i) \leqslant 1
\end{aligned}
$$

for any $i \in S_{0}$, where $B$ is an internal set contained in $A$. Define $\mu(\cdot)$ by

$$
\mu(\cdot)=\frac{\nu(\cdot)}{\operatorname{Cap}_{1}^{(\delta)}(B)} .
$$


Then we have

$$
\begin{aligned}
& \mu\left(S_{0}\right)=1, \quad \mu \in \tau_{0}(\delta) \text { and } \\
& \left.{ }^{\circ} \max _{i \in S_{0}} U_{1}^{(\delta)} \mu(i)\right)={ }^{\circ}\left(\max _{i \in S_{0}} \frac{U_{1}^{(\delta)} \nu(i)}{\operatorname{Cap}_{1}^{(\delta)}(B)}\right) \leqslant\left(\frac{1}{\operatorname{Cap}_{1}^{(\delta)}(B)}\right)<\infty .
\end{aligned}
$$

But

$$
1={ }^{\circ} \mu(B) \leqslant{ }^{\circ} \mu(A) \leqslant 1, \quad \text { i.e., } \quad L(\mu)(A)=1 .
$$

This contradicts to our assumption $L(\mu)(A)=0$. We have proved that

$$
{ }^{\circ} \mathrm{Cap}_{1}^{(\delta)}(A)=0 .
$$

THEOREM 4.5. For $\delta \in T_{\delta}$, we assume that the Dirichlet form $\left(\mathcal{E}^{(\delta)}(\cdot, \cdot), \mathcal{D}\left(\mathcal{E}^{(\delta)}\right)\right)$ has the following property:

$$
\begin{aligned}
\forall A \subset S_{0}, & { }^{\circ} \operatorname{Cap}_{i}^{(\delta)}(A)=\infty \\
& \Rightarrow \exists B \subset A \text { such that } 0<{ }^{\circ} \operatorname{Cap}_{1}^{(\delta)}(B)<\infty .
\end{aligned}
$$

Let $\widehat{\tau}_{0}(\delta)$ be the family of all positive measures of $\delta$-finite energy integrals. Then the following statements are equivalent for $A \in \mathcal{A}\left(\mathcal{S}_{0}\right)$ (in particular, $A \in \sigma\left(\mathcal{S}_{0}\right)$ ):

(1) $A$ is zero $\delta$-capacity, i.e., ${ }^{\circ} \mathrm{Cap}_{1}^{(\delta)}(A)=0$.

(2) For any $\mu \in \widehat{T}_{0}(\delta), L(\mu)(A)=0$.

(3) For any $\mu \in \widehat{\tau}_{00}(\delta), L(\mu)(A)=0$, where

$$
\hat{\tau}_{\infty}(\delta)=\left\{\mu \in \widehat{\tau}_{0}(\delta) \mid \mu\left(S_{0}\right)=1,{ }^{\circ}\left\|U_{1}^{(\delta)} \mu\right\|_{\infty}<\infty\right\} .
$$

Proof. $(1) \Rightarrow(2) \Rightarrow(3)$ is clear by Corollary 4.1 . We can show $(2) \Rightarrow(1)$ and $(3) \Rightarrow(1)$ in the same way as the proof of Theorem 4.4.

\section{Internal Additive Functionals and Associated Measures}

For $\delta \in T$, we have introduced the hyperfinite $m$-symmetric Markov chain $\left(\Omega, X^{(\delta)},\left\{\mathcal{F}_{t}^{(\delta)} \mid t \in T_{\delta}\right\},\left\{P_{i} \mid i \in S\right\}\right)$ in Section 2. The hyperfinite Dirichlet form associated with $X^{(\delta)}$ is given by (2.29) in Section 2 . As in the study of standard Markov processes, we define a family of translation operators $\left\{\theta_{t} \mid t \in T\right\}$ of $\Omega$. That is, for each $t \in T, \theta_{t}$ is a map from $\Omega$ to $\Omega$ defined by

$$
\omega \in \Omega \Rightarrow \theta_{t} \omega \in \Omega \text { and for any } s \in T, \theta_{t} \omega(s)=\omega(s+t) \text {. }
$$


Hence for each $\delta \in T$, we have a family of translation operators $\left\{\theta_{t}^{(\delta)} \mid t \in T_{\delta}\right\}$ induced by $\left\{\theta_{t} \mid t \in T\right\}, \theta_{t}^{(\delta)}=\theta_{t}$ for any $t \in T_{\delta}$. In other words, for each $t \in T_{\delta}, \theta_{t}^{(\delta)}$ is a map from $\Omega$ to $\Omega$ given by

$$
\omega \in \Omega \Rightarrow \theta_{t}^{(\delta)} \omega \in \Omega \text { and for any } s \in T_{\delta}, \theta_{t}^{(\delta)} \omega(s)=\omega(s+t) .
$$

DEFINITION 5.1. For any $\delta \in T$, we call an internal ${ }^{*} \mathbf{R}$-valued function $A(\omega, t)$ or $A_{t}(\omega), t \in T_{\delta}, \omega \in \Omega, \delta$-internal additive functional (abbreviated by $\delta$-IAF) if it satisfies the following two conditions:

(1) For each $t \in T_{\delta}, A_{t}(\omega)$ is nonanticipating with respect to the filtration $\left(\Omega,\left\{\mathcal{F}_{t}^{(\delta)} \mid t \in T_{\delta}\right\}\right)$, i.e., $A_{t}(\cdot)$ is $\mathcal{F}_{t}^{(\delta)}$-measurable.

(2) For each $\omega \in \Omega$, we have

$$
A(\omega, 0)=0, A(\omega, t+s)=A(\omega, s)+A\left(\theta_{s}^{(\delta)} \omega, t\right) \quad \text { for any } t, s \in T_{\delta} .
$$

PROPOSITION 5.1. If $A(\omega, t)$ is a $\delta$-internal additive functional, then there exists a hyperfinite measure $\mu_{\langle A\rangle}$ on $S_{0}$ (not necessarily positive) such that $\mu_{\langle A\rangle}(i)=0$ whenever $m(i)=0$ and for all $n \in{ }^{*} \mathbf{N}, f, h \in H$,

$$
\begin{aligned}
& \int_{S_{0}} h(i) E_{i} \sum_{k=0}^{n} f\left(X^{(\delta)}(k \delta)\right)(A(\omega,(k+1) \delta)-A(\omega, k \delta)) \mathrm{d} m(i) \\
& =\sum_{k=0}^{n} \int_{S_{0}} f(i) E_{i} h\left(X^{(\delta)}(k \delta)\right) \mathrm{d} \mu(i) \delta .
\end{aligned}
$$

Proof. Define

$$
\mu_{\langle A\rangle}(0)=0, \quad \mu_{\langle A\rangle}(i)=\frac{1}{\delta} E_{i} A(\omega, \delta) m(i), \quad 1 \leqslant i \leqslant N .
$$

Then

$$
\begin{aligned}
& \int_{S_{0}} h(i) E_{i} \sum_{k=0}^{n} f\left(X^{(\delta)}(k \delta)\right)(A(\omega,(k+1) \delta)-A(\omega, k \delta)) \mathrm{d} m(i) \\
& =\sum_{k=0}^{n} \int_{S_{0}} h(i) E_{i} f\left(X^{(\delta)}(k \delta, \omega)\right) A\left(\theta_{k \delta}^{(\delta)} \omega, \delta\right) \mathrm{d} m(i) \\
& =\sum_{k=0}^{n} \int_{S_{0}} h(i) E_{i} f(X(k \delta)) E_{X(k \delta)} A(\omega, \delta) \mathrm{d} m(i) \\
& =\sum_{k=0}^{n} \int_{S_{0}} f(i) E_{i} A(\omega, \delta) E_{i} h(X(k \delta)) \mathrm{d} m(i) \\
& =\sum_{k=0}^{n} \int_{S_{0}} f(i) E_{i} h(X(k \delta)) \mathrm{d} \mu(i) \delta
\end{aligned}
$$


PROPOSITION 5.2. Let $\mu$ be a hyperfinite measure on $S_{0}$ satisfying $\mu(i)=0$ whenever $m(i)=0$. Then for each $\delta \in T$, there exists a $\delta-I A F A(\omega, t)$ such that (5.4) hold.

Proof. First of all, let $f(s)=\frac{\mu(s)}{m(s)} 1_{(m(s) \neq 0)}$. For each $u \in H$, we have

$$
\int_{S_{0}} u(s) \mathrm{d} \mu(s)=\int_{S_{0}} u(s) f(s) \mathrm{d} m(s)=\mathcal{E}_{1}^{(\delta)}\left(u, G_{-1}^{(\delta)} f\right) .
$$

Define

$$
\begin{aligned}
& A(\omega, 0)=0 \\
& A(\omega, k \delta)=\delta \sum_{l=1}^{k} f\left(X^{(\delta)}(\omega,(l-1) \delta)\right) \quad \text { for } \quad k \in{ }^{*} \mathbf{N}, \quad k \geqslant 1 .
\end{aligned}
$$

It is easy to verify that $A(\omega, t)$ is a $\delta$-IAF. Moreover, for each $i \in S_{0}$,

$$
\frac{1}{\delta} E_{i} A(\omega, \delta) m(i)=\frac{1}{\delta} E_{i} f\left(X^{(\delta)}(\omega, 0)\right) \delta m(i)=f(i) m(i)=\mu(i) .
$$

Therefore, it follows from the proof of Proposition 5.1 that (5.4) holds.

For $\delta \in T$, let $A(\omega, t)$ be a $\delta$-IAF. Define

$$
e(A)=\frac{1}{2 \delta} E(A(\omega, \delta))^{2} \frac{1}{2 \delta} \int_{S_{0}} E_{i} A_{\delta}^{2} \mathrm{~d} m(i)
$$

We call $e(A)$ the energy of $A$. Furthermore, we define mutual energy e $(A, B)$ for $\delta$-internal additive functionals $A$ and $B$ by

$$
e(A, B)=\frac{1}{2 \delta} E(A(\omega, \delta) B(\omega, \delta))
$$

Let $\Delta A(\omega, k \delta)$ be the forward increment of $A(\omega, t)$ at time $k \delta$, i.e.,

$$
\Delta A(\omega, k \delta)=A(\omega,(k+1) \delta)-A(\omega, k \delta) \text { for } k \in{ }^{*} \mathbf{N}
$$

We define the quadratic variation $[A]: \Omega \times T_{\delta} \rightarrow{ }^{*} \mathbf{R}$ by

$$
\begin{aligned}
& {[A](\omega, 0)=0,} \\
& {[A](\omega, n \delta)=\sum_{k=0}^{n-1}(\Delta A(\omega, k \delta))^{2} \text { for } n \in \in^{*} \mathbf{N}, \quad n>0 .}
\end{aligned}
$$


Because

$$
\begin{aligned}
{[A](\omega,(n+m) \delta) } & =\sum_{k=0}^{n-1}(\Delta A(\omega, k \delta))^{2}+\sum_{k=n}^{n+m-1}(\Delta A(\omega, k \delta))^{2} \\
& =\sum_{k=0}^{n-1}(\Delta A(\omega, k \delta))^{2}+\sum_{k=0}^{m-1}(\Delta A(\omega,(k+n) \delta))^{2} \\
& =\sum_{k=0}^{n-1}(\Delta A(\omega, k \delta))^{2}+\sum_{k=0}^{m-1}\left(\Delta A\left(\theta_{n \delta} \omega, k \delta\right)\right)^{2}
\end{aligned}
$$

$[A]$ is a positive $\delta$-IAF. By Proposition 5.1 and its proof, we know that $\mu_{\langle[A]\rangle}(i)=$ $\frac{1}{\delta} E_{i}(A(\omega, \delta))^{2} m(i)$ is the hyperfinite positive measure associated with $[A]$ in the sense (5.4). We call $\mu_{\langle(A A]\rangle}$ the energy measure of $A$. It is obviously from (5.5) and (5.6) that

$$
e(A)=\frac{1}{2} \mu_{\{[A]\}}\left(S_{0}\right) .
$$

Let $u \in H$. For $\delta \in T$, define a $\delta$-IAF $A^{[u]}(\omega, t)$ by

$$
A^{[u]}(\omega, t)=u\left(X^{(\delta)}(\omega, t)\right)-u\left(X^{(\delta)}(\omega, 0)\right) \quad \text { for } \quad t \in T_{\delta}
$$

Then

$$
\begin{aligned}
e\left(A^{[u]}\right) & =\frac{1}{2 \delta} E\left(A^{[u]}(\omega, \delta)\right)^{2} \\
& =\frac{1}{2 \delta} E\left[u\left(X^{(\delta)}(\omega, \delta)\right)-u\left(X^{(\delta)}(\omega, 0)\right)\right]^{2} \\
& =\frac{1}{2 \delta} \sum_{i=0}^{N} E_{i}\left(u\left(X^{(\delta)}(\omega, \delta)\right)-u(i)\right)^{2} m(i) \\
& =\frac{1}{2 \delta} \sum_{i=0}^{N} \sum_{j=0}^{N}(u(j)-u(i))^{2} q_{i j}^{(\delta)} m(i) \\
& =\frac{1}{2 \delta} \sum_{i=1}^{N} \sum_{j=1}^{N}(u(j)-u(i))^{2} q_{i j}^{(\delta)} m(i)+\frac{1}{2 \delta} \sum_{i=1}^{N}(u(i))^{2} q_{i 0}^{(\delta)} m(i) \\
& =\mathcal{E}^{(\delta)}(u, u)-\frac{1}{2 \delta} \sum_{i=1}^{N}(u(i))^{2} q_{i 0}^{(\delta)} m(i) .
\end{aligned}
$$


THEOREM 5.1. For $u \in H, f \in H$, let $\mu_{\langle u\rangle}(\cdot)$ be the energy measure of $A^{[u]}$. We have

$$
\begin{aligned}
\text { (1) } \begin{aligned}
\mu_{\langle u\rangle}(i) & =\frac{1}{\delta} E_{i}\left(A^{[u]}(\omega, \delta)\right)^{2} m(i) \\
& =\frac{1}{\delta} \sum_{j=0}^{N}(u(j)-u(i))^{2} q_{i j}^{(\delta)} m(i) .
\end{aligned} \\
\text { (2) } \int_{S_{0}} f(s) \mu_{(u)}(\mathrm{d} s)=2 \mathcal{E}^{(\delta)}(u f, u)-\mathcal{E}^{(\delta)}\left(u^{2}, f\right) .
\end{aligned}
$$

Proof. (1) Easy!

(2) On the one hand, we have

$$
\int_{S_{0}} f(s) \mu_{\langle u\rangle}(\mathrm{d} s)=\frac{1}{\delta} \sum_{i=0}^{N} \sum_{j=0}^{N} f(i)(u(j)-u(i))^{2} q_{i j}^{(\delta)} m(i) .
$$

On the other hand, we have

$$
\begin{aligned}
2 \mathcal{E}^{(\delta)}(u f, u)-\mathcal{E}^{(\delta)}\left(u^{2}, f\right) \\
=\frac{2}{\delta} \sum_{i=1}^{N}\left[(u(i))^{2} f(i) m(i)-\sum_{j=1}^{N}(u f)(j) u(i) q_{i j}^{(\delta)} m(i)\right] \\
\quad-\frac{1}{\delta} \sum_{i=1}^{N}\left[(u(i))^{2} f(i) m(i)-\sum_{j=1}^{N}(u(i))^{2} f(j) q_{i j}^{(\delta)} m(i)\right] \\
=\frac{1}{\delta} \sum_{i=1}^{N}(u(i))^{2} f(i) m(i)-\frac{1}{\delta} \sum_{i=1}^{N} \sum_{j=1}^{N}(2 u(j)-u(i)) u(i) f(j) q_{i j}^{(\delta)} m(i) \\
=\frac{1}{\delta} \sum_{i=1}^{N}(u(i))^{2} f(i) m(i)-\frac{1}{\delta} \sum_{i=1}^{N} \sum_{j=1}^{N}(2 u(j)-u(i)) u(i) f(j) q_{j i}^{(\delta)} m(j) \\
=\frac{1}{\delta} \sum_{i=1}^{N} f(i) m(i)\left((u(i))^{2}-\sum_{j=0}^{N}(2 u(i)-u(j)) u(j) q_{i j}^{(\delta)}\right) \\
=\frac{1}{\delta} \sum_{i=1}^{N} \sum_{j=0}^{N} f(i)(u(j)-u(i))^{2} q_{i j}^{(\delta)} m(i) .
\end{aligned}
$$


By (5.15) and (5.16), we get (5.14).

\section{Fukushima's Decomposition Theorem}

6.1. DECOMPOSTTION UNDER INDIVIDUAL PROBABILITY MEASURES $P_{i}$

LEMMA 6.1. For $\delta \in T$, let $\nu$ be a positive measure on $S_{0}$ of $\delta$-hyperfinite energy integral. For any $u \in H, t \in T_{\delta}$ and $\varepsilon>0$, we have

$$
\begin{aligned}
& P_{\nu}\left(\omega \mid \exists s \in T_{\delta}^{t}\left(\left|u\left(X^{(\delta)}(\omega, s)\right)\right| \geqslant \varepsilon\right)\right) \\
& \quad \leqslant \frac{2(1+\delta)^{t / \delta}}{\varepsilon}\left[\mathcal{E}_{1}^{(\delta)}\left(U_{1}^{(\delta)} \nu, U_{1}^{(\delta)} \nu\right) \mathcal{E}_{\mathrm{I}}^{(\delta)}(u, u)\right]^{1 / 2}
\end{aligned}
$$

where $P_{\nu}(\cdot)=\int_{S_{0}} P_{i}(\cdot) \mathrm{d} \nu(i)$.

Proof. Let $A=\left\{i \in S_{0} \mid u(i) \geqslant \varepsilon\right\}$, define

$$
\sigma_{A}^{(\delta)}(\omega)=\min \left\{t \in T_{\delta} \mid X(\omega, t) \in A\right\}
$$

Then we have

$$
\begin{aligned}
& P_{\nu}\left(\omega \mid \exists s \in T_{\delta}, s \leqslant t\left(u\left(X^{(\delta)}(\omega, s)\right) \geqslant \varepsilon\right)\right) \\
& \quad=\int_{S_{0}} P_{i}\left\{\omega \mid(1+\delta)^{-\sigma_{A}^{(\delta)} / \delta} \geqslant(1+\delta)^{-(t / \delta)}\right\} \mathrm{d} \nu(i) \\
& \quad \leqslant \int_{S_{0}} E_{i}\left[(1+\delta)^{-\sigma_{A}^{(\delta)} / \delta}(1+\delta)^{t / \delta}\right] \mathrm{d} \nu(i) \\
& =\{1+\delta)^{t / \delta} \int_{S_{0}} e_{1}^{(\delta)}(A)(i) \mathrm{d} \nu(i) \\
& =(1+\delta)^{t / \delta} \mathcal{E}_{1}^{(\delta)}\left(U_{1}^{(\delta)} \nu, e_{1}^{(\delta)}(A)\right) \\
& \quad \leqslant(1+\delta)^{t / \delta}\left[\mathcal{E}_{1}^{(\delta)}\left(U_{1}^{(\delta)} \nu, U_{1}^{(\delta)} \nu\right) \mathcal{E}_{1}^{(\delta)}\left(e_{1}^{(\delta)}(A), e_{1}^{(\delta)}(A)\right)\right]^{1 / 2} \\
& \quad \leqslant \frac{(1+\delta)^{t / \delta}}{\varepsilon}\left[\mathcal{E}_{1}^{(\delta)}\left(U_{1}^{(\delta)} \nu, U_{1}^{(\delta)} \nu\right) \mathcal{E}_{1}^{(\delta)}(u, u)\right]^{1 / 2},
\end{aligned}
$$

where the reason for the last step holding is

$$
\mathcal{E}_{1}^{(\delta)}\left(e_{1}^{(\delta)}(A), e_{1}^{(\delta)}(A)\right) \leqslant \mathcal{E}_{1}^{(\delta)}\left(\frac{|u|}{\varepsilon}, \frac{|u|}{\varepsilon}\right) \leqslant \frac{1}{\varepsilon^{2}} \mathcal{E}_{1}^{(\delta)}(u, u) .
$$

Hence we can prove Lemma 6.1 by using the same argument to $-u$. 
PROPOSITION 6.1. For $\delta \in T$, assume that $\left(\mathcal{E}^{(\delta)}(\cdot, \cdot), \mathcal{D}\left(\mathcal{E}^{(\delta)}\right)\right)$ satisfies condition (4.27) of Theorem 4.5. Let $u,\left\{u_{n} \mid n \in \mathbf{N}\right\}$ be the elements in $H$ and $\delta \in T$. Suppose that

$$
{ }^{\circ} \mathcal{E}_{1}^{(\delta)}\left(u_{n}-u, u_{n}-u\right) \rightarrow 0 \text { as } n \rightarrow \infty .
$$

Then there exists a subsequence $\left\{u_{n_{k}} \mid k \in \mathbf{N}\right\}$ and $a$-exceptional set $B$ such that for all $i \in S_{0}-B, t \in T_{\tilde{\delta}}^{\mathrm{fin}}$,

$$
\begin{gathered}
L\left(P_{i}\right)\left(u_{n_{k}}\left(X^{(\delta)}(\omega, s)\right) \text { converges uniformly to } u\left(X^{(\delta)}(\omega, s)\right)\right. \\
\text { in } \left.s \text { on } T_{\delta}^{t} \text { as } n \rightarrow \infty\right)=1 .
\end{gathered}
$$

Proof. Let $\left\{n_{k} \mid k \in \mathbf{N}\right\}$ be a subsequence satisfying

$$
{ }^{\circ} \mathcal{E}_{1}^{(\delta)}\left(u_{n_{k}}-u, u_{n_{k}}-u\right) \leqslant 2^{-4 k} .
$$

Set

$$
\Lambda_{k}(t)=\left\{\omega \mid \exists s \in T_{\delta}^{t}\left(\left|u_{n_{k}}\left(X^{(\delta)}(\omega, s)\right)-u\left(X^{(\delta)}(\omega, s)\right)\right| \geqslant 2^{-k}\right)\right\} .
$$

For $\nu \in \widehat{\tau}_{00}(\delta)$, we have from Lemma 6.1 that

$$
{ }^{\circ} P_{\nu}\left(\Lambda_{k}(t)\right) \leqslant{ }^{\circ}\left(e^{t} 2^{-k+1}\left[\mathcal{E}_{1}^{(\delta)}\left(U_{1}^{(\delta)} \nu, U_{1}^{(\delta)} \nu\right)\right]^{1 / 2}\right) .
$$

Hence

$$
\sum_{k=1}^{\infty} L\left(P_{\nu}\right)\left(\Lambda_{k}(t)\right)<\infty
$$

By the Borel-Cantelli lemma, we get

$$
L\left(P_{\nu}\right)\left(\bigcap_{k=1}^{\infty} \bigcup_{l=k}^{\infty} \Lambda_{l}(t)\right)=0 .
$$

Set $\Lambda(t)=\bigcap_{k=1}^{\infty} \bigcup_{l=k}^{\infty} \Lambda_{l}(t)$. From Theorem 4.5, Proposition 3.1 and (6.3) that there exists a $\delta$-exceptional set $B(t)$ such that

$$
L\left(P_{i}\right)(\Lambda(t))=0 \text { for any } i \in S_{0}-B(t) .
$$

Now let us select a countable subset $\left\{t_{n} \mid n \in \mathbf{N}\right\} \subset T_{\delta}$ such that $t_{n} \approx n$. We define $\Lambda=\bigcup_{n=1}^{\infty} \Lambda\left(t_{n}\right), B=\bigcup_{n=1}^{\infty} B\left(t_{n}\right)$. It is easy to see that Proposition 6.1 holds.

LEMMA 6.2. For $\delta \in T$, let $A$ be a $\delta$-IAF and $\mu_{\langle A\rangle}(i)$ be the hyperfinite measure defined by (5.5) in Section 5 . Then for any $v \in H$, we have 


$$
\mathcal{E}^{(\delta)}\left(f_{t}, v\right)=\int_{S_{0}}\left[v(i)-E_{i} v\left(X^{(\delta)}(t)\right)\right] \mathrm{d} \mu_{\langle A\rangle}(i) \text { for all } t \in T_{\delta}
$$

where $f_{t}(i)=E_{i} A(\omega, t)$.

Proof. If $k=1$, then

$$
\begin{aligned}
\mathcal{E}^{(\delta)}\left(f_{\dot{\delta}}, v\right) & =\frac{1}{\delta} \int_{S_{0}}\left(v(i)-Q^{\delta} v(i)\right) f_{\delta}(i) \mathrm{d} m(i) \\
& =\int_{S_{0}}\left(v(i)-E_{i} v\left(X^{(\delta)}(\delta)\right)\right) \mathrm{d} \mu_{(A)}(i) .
\end{aligned}
$$

Assume that (6.4) holds whenever $k \leqslant n$, then

$$
\begin{aligned}
\mathcal{E}^{(\delta)}\left(f_{(n+1) \delta}, v\right)= & \frac{1}{\delta} \int_{S_{0}}\left(v(i)-Q^{\delta} v(i)\right) E_{i} A(\omega,(n+1) \delta) \mathrm{d} m(i) \\
= & \frac{1}{\delta} \int_{S_{0}}\left(v(i)-Q^{\delta} v(i)\right)\left(E_{i} A(\omega, n \delta)\right. \\
& \left.+E_{i} A\left(\theta_{n \delta} \omega, \delta\right)\right) \mathrm{d} m(i) \\
= & \int_{S_{0}}\left(v(i)-E_{i} v\left(X^{(\delta)}(n \delta)\right)\right) \mathrm{d} \mu_{(A\rangle}(i) \\
& +\frac{1}{\delta} \int_{S_{0}}\left(E_{i} v\left(X^{(\delta)}(n \delta)\right)-E_{i} v\left(X^{(\delta)}((n+1) \delta)\right)\right) \\
& \times E_{i} A(\omega, \delta) \mathrm{d} m(i) \\
= & \int_{S_{0}}\left[v(i)-E_{i} v\left(X^{(\delta)}((n+1) \delta)\right)\right] \mathrm{d} \mu_{(A\rangle}(i) .
\end{aligned}
$$

LEMMA 6.3. For $\delta \in T$, let $A(\omega, t)$ be a positive $\delta$-IAF. For all positive measure $\nu$ on $S_{0}$ of $\delta$-hyperfinite energy integral, we have

$$
E_{\nu}\left(A_{t}\right) \leqslant(1+t)\left\|U_{1}^{(\delta)} \nu\right\|_{\infty} \mu_{(A\rangle}\left(S_{0}\right) \quad \text { for all } t \in T_{\delta}
$$

Proof. It follows from Lemma 6.2 that

$$
\begin{aligned}
\mathcal{E}_{\nu} A(\omega, t)= & \int_{S_{0}} E_{i} A(\omega, t) \mathrm{d} \nu(i)=\mathcal{E}_{1}^{(\delta)}\left(U_{1}^{(\delta)} \nu, f_{t}\right) \\
= & \int_{S_{0}}\left[U_{1}^{(\delta)} \nu(i)-E_{i}\left(U_{1}^{(\delta)} \nu\left(X^{(\delta)}(t)\right)\right)\right] \mathrm{d} \mu_{\langle A\rangle}(i) \\
& +\int_{S_{0}} f_{t}(i) U_{1}^{(\delta)} \nu(i) \mathrm{d} m(i) \\
\leqslant & \left\|U_{1}^{(\delta)} \nu\right\|_{\infty}\left[\mu_{\langle A\rangle}\left(S_{0}\right)+\int_{S_{0}} f_{t}(i) \mathrm{d} m(i)\right]
\end{aligned}
$$


Noticing that

$$
\begin{aligned}
\int_{S_{0}} f_{(k+1) \delta}(i) \mathrm{d} m(i) & =\int_{S_{0}} E_{i} A(\omega,(k+1) \delta) \mathrm{d} m(i) \\
& =\int_{S_{0}} E_{i} A(\omega, k \delta) \mathrm{d} m(i)+\int_{S_{0}} E_{i} A\left(\theta_{k \delta} \omega, \delta\right) \mathrm{d} m(i) \\
& \leqslant \int_{S_{0}} E_{i} A(\omega, k \delta) \mathrm{d} m(i)+\int_{S_{0}} E_{i} A(\omega, \delta) \mathrm{d} m(i),
\end{aligned}
$$

we can show

$$
\int_{S_{0}} f_{t}(i) \mathrm{d} m(i) \leqslant t \mu_{\langle A\rangle}\left(S_{0}\right)
$$

From (6.6) and (6.7), we obtain (6.5).

DEFINITION 6.1. An internal process $A: \Omega \times T \rightarrow^{*} \mathbf{R}$ is said to be a martingale with respect to $\left(\Omega, \mathcal{F}_{t}^{(\delta)}, P_{i}, i \in S_{0}\right)$ if $\omega \rightarrow A(\omega, t)$ is $\mathcal{F}_{t}^{(\delta)}$ measurable for all $t \in T_{\delta}$, and for all $s, t \in T_{\delta}, s<t$, and all $B \in \mathcal{F}_{s}^{(\delta)}$,

$$
E_{i}\left(1_{B}\left(A_{t}-A_{s}\right)\right)=0 .
$$

It is easy to see that if $[\omega]_{t}^{(\delta)}$ is the equivalence class of $\omega$ defined by $(2.20)$ in Section 2 , then a nonanticipating process $A(\omega, t)$ is a martingale iff

$$
\sum_{\tilde{\omega} \in[\omega]_{i}^{(\delta)}} \Delta A(\tilde{\omega}, t) P_{i}\{\tilde{\omega}\}=0 .
$$

PROPOSITION 6.2. For $\delta \in T$, let $A,\left\{A_{n} \mid n \in \mathbf{N}\right\}$ be $\delta$-internal additive functionals. Assume that ${ }^{\circ} e\left(A_{n}-A\right) \rightarrow 0$ as $n \rightarrow \infty$, and for each $i \in S_{0},\left\{\Omega, \mathcal{F}_{t}^{(\delta)}, A(t)\right.$, $\left.A_{n}(t), P_{i}\right\}$ are martingales for all $n \geqslant 1$. There exists a subsequence $\left\{A_{n_{k}}(t) \mid k \in\right.$ N\} and a $\delta$-exceptional set $B$ such that for all $i \in S_{0}-B, t \in T_{\delta}^{\text {fin }}$,

$$
L\left(P_{i}\right)\left(\omega \mid A_{n_{k}}(\omega, s) \rightarrow A(\omega, s) \text { uniformly on } T_{\delta}^{t}\right)=1
$$

Proof. By (5.10) in Section 5, we know

$$
{ }^{\circ} \mu_{\left\langle\left[A_{n 2}-A\right]\right\rangle}\left(S_{0}\right)={ }^{\circ}\left[2 e\left(A_{n}-A\right)\right] \rightarrow 0 \text { as } n \rightarrow \infty
$$

For simplicity, we suppose that ${ }^{\circ} \mu_{\left\langle\left[A_{n}-A\right]\right\rangle}\left(S_{0}\right)<2^{-3 k}$ (taking a subsequence if necessary). Since $A, A_{n}, n \geqslant 1$ are martingales with respect to $P_{i}, i \in S_{0}$, we get from Lemma 6.3 that for all $\nu \in \tau_{00}(\delta), t \in T_{\delta}^{\text {fin }}$ :

$$
\begin{aligned}
{ }^{\circ}\left[E_{\nu}\left(A_{n}(t)-A(t)\right)^{2}\right] & ={ }^{\circ}\left(E_{\nu}\left(\left[A_{n}-A\right](t)\right)\right) \\
& \leqslant{ }^{\circ}\left[(1+t)\left\|U_{1}^{(\delta)} \nu\right\|_{\infty} 2^{-3 k}\right] .
\end{aligned}
$$


From (6.11) and Doob's inequality (refer to $[3,4.2 .8]$ ), we get

$$
\begin{aligned}
& { }^{\circ} P_{\nu}\left(\max _{s \leqslant t}\left|A_{n}(s)-A(s)\right| \geqslant 2^{-k}\right) \leqslant{ }^{\circ}\left[2^{2 k} E_{\nu}\left(\max _{s \leqslant t}\left|A_{n}(s)-A(s)\right|\right)^{2}\right] \\
& \leqslant{ }^{\circ}\left[2^{2 k+2} E_{\nu}\left(A_{n}(t)-A(t)\right)^{2}\right] \leqslant{ }^{\circ}\left[4(1+t)\left\|U_{1}^{(\delta)} \nu\right\|_{\infty} 2^{-k}\right] .
\end{aligned}
$$

It is easy to show Proposition 6.2 by using Borel-Cantelli lemma, Theorem 4.4 and Theorem 3.2 .

DEFINITION 6.2. (1) We call an internal function in $H S$-bounded if there is a positive constant $C$ such that $|u(i)| \leqslant C$ for all $i \in S_{0}$.

(2) An internal function $f: T_{\delta} \rightarrow{ }^{*} \mathbf{R}$ is called $S$-continuous if $f(s) \approx f(t)$ whenever $s \approx t$ and $s$ and $t$ are nearstandard.

LEMMA 6.4. For $\delta \in T, u \in \operatorname{Fin}(H)$, define a $\delta$-IAF $A^{\delta}$ by

$$
\begin{aligned}
& A^{\delta}(\omega, 0)=0, \\
& A^{\delta}(\omega, n \delta)=\delta \sum_{k=1}^{n} u(X(\omega,(k-1) \delta)), \quad n \in \mathbf{N}, \quad n \geqslant 1 .
\end{aligned}
$$

Then there exists a properly $\delta$-exceptional set $B \subset S_{0}$ such that for all $i \in S_{0}-B$,

$$
L\left(P_{i}\right)\left\{\omega \mid A^{\delta}(\omega, \cdot) \quad \text { is } S \text {-continuous }\right\}=1 \text {. }
$$

Proof. First of all, we assume that $u$ is $S$-bounded. We have for any $\omega \in \Omega$,

$$
\left|A^{\delta}(\omega, t)-A^{\delta}(\omega, s)\right| \leqslant t-s \mid\left(\max _{i \in S_{0}}|u(i)|+1\right) .
$$

This implies that $A^{\delta}(\omega, t)$ is $S$-continuous.

Next, we suppose that $u \in \operatorname{Fin}(H)$. For each $n$, denote

$$
B_{n}=\left\{s \in S_{0}|| u(s) \mid \geqslant n\right\}
$$

and define

$$
\sigma_{B_{n}}(\omega)=\min \left\{t \in T_{\delta} \mid X(t) \in B_{n}\right\} .
$$

Then

$$
\begin{aligned}
& P\left(\omega \mid \exists t \in T_{\delta}^{1}\left(X(\omega, t) \in B_{n}\right)\right) \\
& \quad=P\left(\omega \mid \sigma B_{n}(\omega) \leqslant 1\right) \\
& \quad \leqslant n^{-2} \int_{S_{0}} E_{i}\left[u\left(X\left(\sigma_{B_{n}}\right)\right)\right]^{2} \mathrm{~d} m(i) \\
& \quad \leqslant n^{-2} \int_{S_{0}}[u(i)]^{2} \mathrm{~d} m(i) .
\end{aligned}
$$


Therefore, we get

$$
L(P)\left(\omega \mid \exists t \in T_{\delta}^{1}\left(X(\omega, t) \in \bigcap_{n=1}^{\infty} B_{n}\right)\right)=0 .
$$

This implies that $\left\{\left.s \in S_{0}\right|^{\circ} \mid u(s)=\infty\right\}$ is a $\delta$-exceptional set. Let $B$ be a properly $\delta$-exceptional set containing $\left\{\left.s \in S_{0}\right|^{\circ}|u(s)|=\infty\right\}$ (Proposition 3.1). For each $n \in \mathbf{N}$, define

$$
\sigma_{n}(\omega)=\min \left\{t \in T_{\delta}|| u(X(\omega, t)) \mid \geqslant n\right\} .
$$

Then for each $\omega \in \Omega, A^{\delta}(\omega, \cdot)$ is continuous in $\left[0, \sigma_{n}(\omega)\right)$ for every $n$.

Moreover, for each $i \in S_{0}-B$, we have

$$
L\left(P_{i}\right)\left\{\left.\omega\right|^{\circ} \sigma_{n}(\omega) \uparrow \infty \text { as } n \rightarrow \infty\right\}=1 .
$$

We have shown Lemma 6.4 .

THEOREM 6.1. For $\delta \in T$, assume $\left(\mathcal{E}^{(\delta)}(\cdot, \cdot), \mathcal{D}\left(\mathcal{E}^{(\delta)}\right)\right)$ satisfies $(4.27)$ in Section 4. For any $u \in \mathcal{D}\left(\mathcal{E}^{(\delta)}\right)$, there are two $\delta$-internal additive functionals $M^{[u]}(\omega, t)$ and $N^{[u]}(\omega, t)$ such that

(1) $A^{[u]}(\omega, t)=M^{[u]}(\omega, t)+N^{[u]}(\omega, t)$.

(2) For each $i \in S_{0}, M^{[u]}$ is a martingale with respect to $\left(\Omega,\left\{\mathcal{F}_{t}^{(\delta)}\right\}, P_{i}\right)$.

(3) $e\left(N^{[u]}\right) \approx 0$ and $E\left[N^{[u]}\right](t) \approx 0$ for all $t \in T_{\delta}^{\text {fin }}$.

(4) There exists a $\delta$-exceptional set $A \subset S_{0}$ such that for all $i \in S_{0}-A$,

$$
\begin{aligned}
& { }^{\circ} E_{i}\left(M^{[u]}(\omega, t)\right)^{2}<\infty \text { for all } t \in T_{\delta}^{\mathrm{fin}}, \\
& L\left(P_{i}\right)\left(\omega \mid N^{[u]}(\omega, \cdot) \quad \text { is } S \text {-continuous }\right)=1 .
\end{aligned}
$$

Proof. Define

$$
\begin{aligned}
& N^{[u]}(\omega, 0)=0, \\
& \Delta N^{[u]}(\omega, t)=Q^{\delta} u\left(X^{(\delta)}(\omega, t)\right)-u\left(X^{(\delta)}(\omega, t)\right), \quad t \in T_{\delta},
\end{aligned}
$$

and

$$
M^{[u]}(\omega, t)=u\left(X^{(\delta)}(\omega, t)\right)-u\left(X^{(\delta)}(\omega, 0)\right)-N^{[u]}(\omega, t) .
$$

For each $t \in T_{\delta}$, we have

$$
\begin{aligned}
& M^{[u]}(\omega, t+\delta)-M^{[u]}(\omega, t) \\
& \quad=u\left(X^{(\delta)}(\omega, t+\delta)\right)-Q^{\delta} u\left(X^{(\delta)}(\omega, t)\right) .
\end{aligned}
$$


It is easy to see from (6.9) and (6.18) that $\left(\Omega, \mathcal{F}_{t}^{(\delta)}, M^{[u]}(\omega, t), P_{i}\right)$ is a martingale for each $i \in S_{0}$. Furthermore, have

$$
\begin{aligned}
\frac{1}{\delta} E\left(\Delta N^{[u]}(t)\right)^{2} & \leqslant \frac{1}{\delta} \sum_{i=1}^{N}\left(Q^{\delta} u(i)-u(i)\right)^{2} m(i) \\
& =\mathcal{E}^{(\delta)}\left(u, u-Q^{\delta} u\right) \approx 0 .
\end{aligned}
$$

From (6.19), we obtain

$$
E\left[N^{[u]}\right](t) \leqslant t \mathcal{E}^{(\delta)}\left(u, u-Q^{\delta} u\right) \approx 0 \text { for each } t \in T_{\delta}^{\text {fin }},
$$

and

$$
e\left(N^{[u]}\right)=\frac{1}{2 \delta} E\left(N^{[u]}(\omega, \delta)\right)^{2} \leqslant \frac{1}{2} \mathcal{E}\left(u, u-Q^{\delta} u\right) \approx 0 .
$$

Therefore, we deduce from (5.12) in Section 5 and (6.20) that

$$
e\left(M^{[u]}\right)=e\left(A^{[u]}-N^{[u]}\right) \approx e\left(A^{[u]}\right) \leqslant \mathcal{E}^{(\delta)}(u, u) .
$$

By using Lemma 6.3 and (5.10) in Section 5 and (6.21), we get for any $\nu \in \tau_{00}(\delta)$, $t \in T_{\delta}^{\text {fin }}$

$$
\begin{aligned}
& { }^{\circ}\left(E_{\nu}\left(M^{[u]}(\omega, t)\right)^{2}\right) \\
& \quad={ }^{\circ}\left(E_{\nu}\left[M^{[u]}\right](t)\right) \leqslant{ }^{\circ}\left[(1+t)\left\|U_{1}^{(\delta)} \nu\right\|_{\infty} \mu_{\left\langle\left[M^{[u]}\right]\right\rangle}\left(S_{0}\right)\right] \\
& \quad={ }^{\circ}\left[(1+t)\left\|U_{1}^{(\delta)} \nu\right\|_{\infty} 2 e\left(M^{[u]}\right)\right] \\
& \quad \leqslant{ }^{\circ}\left[(1+t)\left\|U_{1}^{(\delta)} \nu\right\|_{\infty} 2 \mathcal{E}^{(\delta)}(u, u)\right]<\infty .
\end{aligned}
$$

Therefore, it follows from Theorem 4.4 and (6.22) that there exists a $\delta$-zero capacity set $A_{1} \subset S_{0}$ such that

$$
{ }^{\circ} E_{i}\left(M^{[u]}(\omega, t)\right)^{2}<\infty \text { for all } t \in T_{\delta}^{\text {fin }}, \quad i \in S_{0}-A_{1} .
$$

Put $u_{n}=Q^{1 / n} u, n \in \mathbf{N}$, then

$$
\begin{aligned}
\Delta N^{\left[u_{n}\right]}(\omega, t) & =Q^{\delta} u_{n}(X(\omega, t))-u_{n}(X(\omega, t)) \\
& =-\delta A^{(\delta)} u_{n}(X(\omega, t)) .
\end{aligned}
$$

First of all, we have

$$
\begin{aligned}
& \int_{S_{0}}\left(A^{(\delta)} u_{n}(i)\right)^{2} \mathrm{~d} m(i) \\
& \quad=\mathcal{E}^{(\delta)}\left(u_{n}, A^{(\delta)} u_{n}\right)=\mathcal{E}^{(\delta)}\left(u_{n},\left(u_{n}-Q^{\delta} u_{n}\right)\right) \frac{1}{\delta}
\end{aligned}
$$


By the proof of ([3], 5.1.4 Proposition and 5.1.7 Lemma), we have

$$
\begin{aligned}
& \mathcal{E}^{(\delta)}\left(u_{n}, u_{n}-Q^{\delta} u_{n}\right) \\
& \quad \leqslant \mathcal{E}^{(\delta)}\left(u_{n}, u_{n}\right)-\mathcal{E}^{(\delta)}\left(Q^{\delta} u_{n}, Q^{\delta} u_{n}\right) \leqslant n \delta \mathcal{E}^{(\delta)}(u, u) .
\end{aligned}
$$

Thus we get from (6.25) and (6.26) that

$$
\int_{S_{0}}\left(A^{(\delta)} u_{n}(i)\right)^{2} \mathrm{~d} m(i) \leqslant{ }^{\circ}\left(n \mathcal{E}^{(\delta)}(u, u)\right)<\infty .
$$

Therefore, we know from (6.27) and Lemma 6.4, for each $n \in \mathbf{N}$, there exists a properly $\delta$-exceptional set $B_{n} \subset S_{0}$ such that for all $i \in S_{0}-B_{n}$,

$$
L\left(P_{i}\right)\left\{\omega \mid N^{\left[u_{n}\right]}(\omega, \cdot) \quad \text { is } S \text {-continuous }\right\}=1 .
$$

It follows from (6.21) that

$$
{ }^{\circ} e\left(M^{[u]}-M^{\left[u_{n}\right]}\right) \leqslant{ }^{\circ} \mathcal{E}^{(\delta)}\left(u-u_{n}, u-u_{n}\right) \rightarrow 0 \text { as } n \rightarrow \infty .
$$

(6.29) and Proposition 6.1 and Proposition 6.2 imply that there exist a subsequence $\left\{n_{k} \mid k \in \mathbf{N}\right\}$ and a $\delta$-exceptional set $B$ such that

$$
L\left(P_{i}\right)\left(\Omega_{0}\right)=1 \text { for all } i \in S_{0}-B,
$$

where set $\Omega_{0}=\left\{\omega \in \Omega \mid u_{n_{k}}\left(X^{(\delta)}(\omega, s)\right)\right.$ and $M^{\left[u_{n_{k}}\right]}(\omega, s)$ converges uniformly to $u\left(X^{\{\delta)}(\omega, s)\right)$ and $M^{[u]}(\omega, s)$ on each $S$-bounded subset of $T_{\delta}$, respectively . Set $A=B \cup A_{1} \cup\left(\cup_{n=1}^{\infty} B_{n}\right)$, then we see Theorem 6.1 (4) hold from (6.23), (6.28), (6.30) and Theorem 3.2.

\subsection{DECOMPOSITION UNDER WHOLE MEASURE $P$}

In the following, we shall consider similar decomposition as Theorem 6.1 under the whole measure $P$. We will see that we may work under quite weak conditions. Just as Lemma 6.1, we have

LEMMA 6.5. Let $X$ be a hyperfinite Markov chain and $\mathcal{E}(\cdot, \cdot)$ its Dirichlet form. For $\delta \in T$, all $u \in H, t \in T_{\delta}$ and $\varepsilon>0$, we have

$$
P\left(\omega \mid \exists s \in T_{\delta}^{t}\left(\left|u\left(X^{(\delta)}(\omega, s)\right)\right| \geqslant \varepsilon\right)\right) \leqslant \frac{2(1+\delta)^{t / \delta}}{\varepsilon^{2}} \mathcal{E}_{1}^{(\delta)}(u, u) .
$$

REMARK 6.1.The proof of this result can be found in [3], 5.3.6 Proposition, p. 257.

COROLLARY 6.1. Let $u, u_{n}, n \in \mathbf{N}$ be elements in $H$, and assume that 


$$
{ }^{\circ} \mathcal{E}_{1}^{(\delta)}\left(u-u_{n}, u-u_{n}\right) \rightarrow 0 \text { as } n \rightarrow \infty
$$

There is a subsequence $\left\{u_{n_{k}}\right\}$ such that for a.e. $\omega, u_{n_{k}}\left(X^{(\delta)}\right)$ converges uniformly to $u\left(X^{(\delta)}\right)$ on all $S$-bounded subsets of $T$.

Proof. By Lemma 6.5 and basic measure theory.

DEFINITION 6.3. A Dirichlet form $\mathcal{E}(\cdot, \cdot)$ is normal with respect to $\delta \in T$ if

$$
\left\langle Q^{\delta} u, u\right\rangle \geqslant 0 \text {. }
$$

THEOREM 6.2. For $\delta \in T$, let us assume that $\left(\mathcal{E}^{(\delta)}(\cdot, \cdot), \mathcal{D}\left(\mathcal{E}^{(\delta)}\right)\right)$ is a normal, hyperfinite Dirichlet form. For any $u \in \mathcal{D}\left(\mathcal{E}^{(\delta)}\right)$, there are two $\delta$-internal additive functionals $M^{[u]}(\omega, t)$ and $N^{[u]}(\omega, t)$ such that

(1) $A^{[u]}(\omega, t)=M^{[u]}(\omega, t)+N^{[u]}(\omega, t)$.

(2) $M^{[u]}$ is a $\lambda^{2}$-martingale with respect to $\left(\Omega,\left\{\mathcal{F}_{t}^{(\delta)}\right\}, P\right)$.

(3) $N^{[u]}$ is $S$-continuous and $E\left[N^{[u]}\right](t) \approx 0$ for all $t \in T_{\delta}^{\text {fin }}$.

Proof. The proof of this result is something like that of Theorem 6.1. We remind that the detail demonstration was given in [3]. Hence we do not discuss it so carefully here.

\section{Internal Multiplicative Functionals}

\subsection{INTERNAL MULTIPLICATIVE FUNCTIONAL}

DEFINITION 7.1. For $\delta \in T$, an internal function $M(\omega, t), t \in T_{\delta}, \omega \in \Omega$, is said to be a $\delta$-internal multiplicative functional (abbreviated by $\delta$-IMF) of $X^{(\delta)}(\omega, t)$ iff

(i) For each $t \in T_{\delta}, M_{t}(\cdot)$ is $\mathcal{F}_{t}^{(\delta)}$-measurable.

(ii) For each $t \in T_{\delta}, \omega \in \Omega, M(\omega, t) \in{ }^{*}[0,1]$.

(iii) For each $\omega \in \Omega$, we have

$$
M(\omega, 0)=1, \quad M(\omega, t+s)=M(\omega, s) M\left(\theta_{s}^{(\delta)} \omega, t\right), \forall t, s \in T_{\delta} .
$$

REMARK 7.1.Let $A(\omega, t)$ be a non-negative $\delta$-internal additive functional. We can define a $\delta$-MMF $M(\omega, t)$ by

$$
M(\omega, t)=\exp (-A(\omega, t)) \quad \text { for all } \omega \in \Omega, \quad t \in T_{\delta} .
$$

It $M(\omega, t)$ is a $\delta$-internal multiplicative functional, let us define a family of operators $\left\{P^{t} \mid t \in T_{\delta}\right\}$ on $H$ by

$$
P^{t} f(i)=E_{i}\left\{f\left(X^{(\delta)}(\omega, t)\right) M(\omega, t)\right\} .
$$


Then we have for all $t, s \in T_{\delta}, i \in S$,

$$
\begin{aligned}
P^{t+s} f(i) & =E_{i}\left\{f\left(X^{(\delta)}(\omega, t+s)\right) M(\omega, t+s)\right\} \\
& =E_{i}\left\{f\left(X^{(\delta)}\left(\theta_{s}^{(\delta)} \omega, t\right)\right) M(\omega, s) M\left(\theta_{s}^{(\delta)} \omega, t\right)\right\} \\
& =E_{i}\left\{M(\omega, s) E_{X^{(\delta)}(\omega, s)}\left[f\left(X^{(\delta)}(t)\right) M_{t}\right]\right\} \\
& =P^{s} P^{t} f(i) .
\end{aligned}
$$

Hence, $\left\{P^{t} \mid t \in T_{\delta}\right\}$ is a semigroup. We call it the semigroup generated by $\left(X^{(\delta)}, M\right)$. Moreover, we have for all $f \in H$,

$$
P^{0} f(i)=E_{i}\left[f\left(X^{(\delta)}(\omega, 0)\right) M(\omega, 0)\right]=f(i) .
$$

In particular, we have

$$
P^{0} 1=1
$$

Since $M(\omega, \delta)$ is $\mathcal{F}_{\delta}^{(\delta)}$-measurable, we have

$$
M(\omega, \delta)=\sum_{i, j=0}^{N} 1_{[\omega](i j)}(\omega) M_{i j},
$$

where $[\hat{\omega}](i j)=\{\omega \mid \omega(0)=i$ and $\omega(\delta)=j\}$ and $\left\{M_{i j} \mid i, j=0,1,2, \ldots, N\right\}$ is a family of positive hyperreals. Moreover, $M_{i j} \in{ }^{*}[0,1]$. Therefore, the transition matrix $\left\{p_{i j}^{(\delta)} \mid i, j=0,1,2, \ldots, N\right\}$ of $\left\{P^{t} \mid t \in T_{\delta}\right\}$ is given by

$$
p_{i j}^{(\delta)}=q_{i j}^{(\delta)} M_{i j}, \quad M_{i j} \in^{*}[0,1], \quad i, j=0,1,2, \ldots, N .
$$

From (7.8), we know for all non-negative internal functions $f \in H$,

$$
P^{t} f(i) \leqslant Q^{t} f(i), \quad \forall i \in S, \quad t \in T_{\delta} .
$$

\subsection{SUBORDINATE SEMIGROUP}

DEFINITION 7.2. A semigroup $\left\{P^{t} \mid t \in T_{\delta}\right\}$ of positive linear operators from $H$ to $H$ is said to be subordinate to $\left\{Q^{t} \mid t \in T_{\delta}\right\}$ iff $P^{t} f(i) \leqslant Q^{t} f(i)$ for all $t \in T_{\delta}, f \in H, f(i) \geqslant 0, i \in S$ and $P^{0}=I$.

THEOREM 7.1. Let $\left\{P^{t} \mid t \in T_{\delta}\right\}$ be a semigroup on $H$. Then the following two conditions are equivalent:

(1) $\left\{P^{t} \mid t \in T_{\delta}\right\}$ is subordinate to $\left\{Q^{t} \mid t \in T_{\delta}\right\}$.

(2) There exists a $\delta$-IMF $M(\omega, t)$ of $X^{(\delta)}(\omega, t)$ generating $\left\{P^{t} \mid t \in T_{\delta}\right\}$. 
Proof. (2) $\Rightarrow$ (1). It follows from (7.6) and (7.9).

$(1) \Rightarrow(2)$. Let $\left\{p_{i j}^{(\delta)} \mid i, j=0,1,2, \ldots, N\right\}$ be the transition matrix of semigroup $\left\{P^{t} \mid t \in T_{\delta}\right\}$.Then for each $f \in H$, we have

$$
\begin{aligned}
& Q^{\delta} f(i)=\sum_{j=1}^{N} q_{i j}^{(\delta)} f(j), \\
& P^{\delta} f(i)=\sum_{j=1}^{N} p_{i j}^{(\delta)} f(j) .
\end{aligned}
$$

Since $\left\{P^{t} \mid t \in T_{\delta}\right\}$ is subordinate to $\left\{Q^{t} \mid t \in T_{\delta}\right\}$, we can see

$$
p_{i j}^{(\delta)} \leqslant q_{i j}^{(\delta)}, \forall i, \quad j=0,1,2, \ldots, N .
$$

Define

$$
\widehat{M}(i, j)=\frac{p_{i j}^{(\delta)}}{q_{i j}^{(\delta)}} 1_{\left(q_{i j}^{(\delta)} \neq 0\right)}
$$

where we define $\frac{a}{0}=0, a \in{ }^{*}[0, \infty)$. Put

$$
M(\omega, 0)=1, M(\omega, \delta)=\widehat{M}\left(X^{(\delta)}(\omega, 0), X^{(\delta)}(\omega, \delta)\right)
$$

For all $i \in S$, we have for any $f \in H$,

$$
E_{i}\left[f\left(X^{(\delta)}(\omega, \delta)\right) M(\omega, \delta)\right]=\sum_{j=1}^{N} f(j) \widehat{M}(i, j) q_{i j}^{(\delta)}=P^{\delta} f(i) .
$$

By using mathematical deduction, we define

$$
M(\omega,(k+1) \delta)=M(\omega, k \delta) M\left(\theta_{k \delta}^{(\delta)} \omega, \delta\right) \quad \text { for all } \quad k \in{ }^{*} \mathbf{N} .
$$

It is easy to show that $M(\omega, t)$ is a $\delta$-IMF generating $\left\{P^{t} \mid t \in T_{\delta}\right\}$.

\subsection{SUBPROCESSES}

In Section 2 we define $m$-symmetric Markov process $X^{(\delta)}(\omega, t)$ associated with the hyperfinite Dirichlet form $\mathcal{E}^{(\delta)}(\cdot, \cdot)$. Let $\left\{\widehat{\Omega}, \widehat{\mathcal{F}}_{t}^{(\delta)}, Y^{(\delta)}(\hat{\omega}, t), \widehat{\theta}_{t}^{(\delta)}, \widehat{P}_{i}\right\}_{t \in T_{\delta}}$ be a Markov process with state space $(S, S)$. We call $Y^{(\delta)}(\hat{\omega}, t)$ a subprocess of $X^{(\delta)}(\omega, t)$ iff the semigroup $\left\{\widehat{P}^{t} \mid t \in T_{\delta}\right\}$ of $Y^{(\delta)}(\hat{\omega}, t)$ is subordinate to $\left\{Q^{t} \mid t \in T_{\delta}\right\}$.

Let $Y^{(\delta)}(\widehat{\omega}, t)$ be a subprocess of $X^{(\delta)}(\omega, t)$. From Theorem 7.1, there exists a $\delta$-IMF $M(\omega, t)$ of $X^{(\delta)}(\omega, t)$ such that

$$
\widehat{E}_{i} f\left(Y^{(\delta)}(\widehat{\omega}, t)\right)=E_{i}\left[f\left(X^{(\delta)}(\omega, t)\right) M(\omega, t)\right] \quad \text { for all } t \in T_{\delta}
$$


where $\widehat{E}_{i}(\cdot)$ is the expectation operator corresponding to $Y^{(\delta)}(\hat{\omega}, t)$.

Now we are interested in the following question. Given a $\delta-\operatorname{MMF} M(\omega, t)$, could we construct a subprocess $Y^{(\delta)}(\omega, t)$ such that $(7.17)$ holds? The answer is yes! In fact, let $\widehat{\Omega}=\Omega, \hat{\mathcal{F}}_{t}^{(\delta)}=\mathcal{F}_{t}^{(\delta)}, Y^{(\delta)}(\omega, t)=X^{(\delta)}(\omega, t), \hat{\theta}_{t}^{(\delta)}=\theta_{t}^{(\delta)}$. Furthermore, let $\left\{P^{t} \mid t \in T_{\delta}\right\}$ be the semigroup given by (7.4), and $\left\{p_{i j}^{(\delta)} \mid i, j=0,1,2, \ldots\right\}$ be the transition matrix of $\left\{P^{t} \mid t \in T_{\delta}\right\}$. Define for $\omega \in \widehat{\Omega}=\Omega, k \in{ }^{*} \mathbf{N}$,

$$
\hat{P}_{i}\left([\omega]_{k \delta}\right)=\delta_{i \omega(0)} \prod_{n=0}^{k-1} p(\omega(n \delta), \omega((n+1) \delta)) .
$$

It is obviously that (7.17) holds with respect to $Y^{(\delta)}(\omega, t)$. We call $Y^{(\delta)}(\omega, t)$ the canonical subprocess associated with $\left(X^{(\delta)}, M\right)$. We notice that $\left\{p_{i j}^{(\delta)} \mid i, j=\right.$ $0,1,2, \ldots, N\}$ need not have the regularities (2.1) and (2.2) in Section 2 .

\subsection{FEYNMAN-KAC FORMULAE}

Let $\left\{q_{i}^{(\delta)} \mid i=1,2, \ldots, N\right\}$ be a $1 \times N$ matrix satisfying

$$
0 \leqslant q_{i}^{(\delta)} \leqslant q_{i i}^{(\delta)}, \quad i=1,2, \ldots, N .
$$

Define a transition matrix $P^{(\delta)}=\left\{p_{i j}^{(\delta)} \mid i, j=0,1,2, \ldots, N\right\}$ by

$$
\begin{aligned}
& p_{i j}^{(\delta)}=q_{i j}^{(\delta)}-q_{i}^{(\delta)} \delta_{i j} \text { for } i, j=1,2, \ldots, N, \\
& p_{i 0}^{(\delta)}=q_{i 0}^{(\delta)}+q_{i}^{(\delta)} \text { for } i=1,2, \ldots, N \text { and } \\
& p_{00}^{(\delta)}=1, \quad p_{i 0}^{(\delta)}=0 \text { for } i=1,2, \ldots, N .
\end{aligned}
$$

Let $\left\{P^{t} \mid t \in T_{\delta}\right\}$ be the semigroup with $P^{(\delta)}=\left\{p_{i j}^{(\delta)} \mid i, j=0,1,2, \ldots, N\right\}$ as its transition matrix. Then we have

THEOREM 7.2.(i) The semigroup $\left\{P^{t} \mid t \in T_{\delta}\right\}$ is symmetric with respect to $m$.

(ii) The Dirichlet form associated with $P^{(\delta)}$ and $m$ is given by

$$
\widehat{\mathcal{E}}^{(\delta)}(u, v)=\mathcal{E}^{(\delta)}(u, v)+\sum_{i=1}^{N} u(i) v(i) q_{i}^{(\delta)} m(i) .
$$

(iii) There exists a $\delta$-IMF $M(\omega, t)$ such that for any $f \in H, i \in S, t \in T_{\delta}$,

$$
P^{t} f(i)=E_{i}\left[f\left(X^{(\delta)}(\omega, t)\right) M(\omega, t)\right]
$$

Proof. (i) and (ii) are obvious. (iii) is followed from Theorem 7.1. 


\section{Transformation of Hyperfinite Dirichlet Forms}

In this section, we assume that

$$
q_{i i}=0 \text { for all } i=1,2, \ldots, N .
$$

In fact, this assumption will not affect our theory. The reason comes from the proof of $[3,5.3 .3$ Proposition]. Actually, for general Dirichlet form $\mathcal{E}(\cdot, \cdot)$ in (2.17) in Section 2, we define

$$
\widehat{m}(i)=\left(1-q_{i i}\right) m(i), \quad \widehat{q}_{i i}=0 \quad \text { for all } i=1,2, \ldots, N .
$$

Moreover, if $i=1,2, \ldots, N$ and $q_{i i}<1$, define

$$
\widehat{q}_{i j}=\frac{q_{i j}}{1-q_{i i}} \text { for } j \neq i, j \in\{0,1,2, \ldots, N\}
$$

if $i=1,2, \ldots, N$ and $q_{i i}=1$, define

$$
\widehat{q}_{i j}=0 \text { for } j \neq 0, i \text { and } \widehat{q}_{i 0}=1 \text {. }
$$

Besides, let $\widehat{q}_{00}=1, \widehat{q}_{0 j}=0, j=1,2, \ldots, N$. It is very easy to verify from Beurling-Deny formulae that the Dirichlet form associated with $\hat{m}$ and $\hat{Q}=$ $\left\{\widehat{q}_{i j} \mid i, j=0,1,2, \ldots, N\right\}$ is $\mathcal{E}(\cdot, \cdot)$.

Let $\Phi$ be an internal nonnegative function in $H$. We define a quadratic form in the following way

$$
\mathcal{E}^{\Phi}(u, v)=\frac{1}{\Delta t} \sum_{1 \leqslant i \leqslant j \leqslant N}(u(i)-u(j))(v(i)-v(j)) \Phi(i) \Phi(j) q_{i j} m(i) .
$$

It is easy to see that $\mathcal{E}^{\Phi}(\cdot, \cdot)$ has Markov property defined in Section 2 . Thus by $\left[3,5.3 .3\right.$ Proposition] there exists a transition matrix $\widehat{P}=\left\{\widehat{p}_{i j} \mid 0 \leqslant i, j \leqslant N\right\}$ and a symmetric measure $\widehat{m}(\cdot)$ such that

$$
\mathcal{E}^{\Phi}(u, v)=\int_{S_{0}} u(i)\left(v(i)-\hat{P}^{\Delta t} v(i)\right) \frac{1}{\Delta t} \mathrm{~d} \widehat{m}(i)
$$

In the following, we will find the $\widehat{P}$ and $\widehat{m}$. From (8.3) and the Beurling-Deny formulae, we have

$$
\begin{aligned}
\mathcal{E}^{\Phi}(u, v)= & \frac{1}{\Delta t}\left[\sum_{1 \leqslant i<j \leqslant N}(u(i)-u(j))(v(i)-v(j)) \hat{p}_{i j} \hat{m}(i)\right. \\
& \left.+\sum_{i=1}^{N} u(i) v(i) \widehat{p}_{i 0} \widehat{m}(i)\right] .
\end{aligned}
$$


Comparing (8.2) and (8.4), we must have

$$
\begin{aligned}
& \Phi(i) \Phi(j) q_{i j} m(i)=\widehat{p}_{i j} \widehat{m}(i) \text { for all } 1 \leqslant i, j \leqslant N, \\
& \hat{p}_{i 0} \hat{m}(i)=0 \text { for all } i=1,2, \ldots, N .
\end{aligned}
$$

Therefore, we get

$$
\begin{aligned}
\hat{m}(i) & =\sum_{j=0}^{N} \widehat{p}_{i j} \hat{m}(i)=\sum_{j \neq i} \Phi(j) q_{i j} \Phi(i) m(i)+\widehat{p}_{i i} \hat{m}(i) \\
& =E_{i}[\Phi(X(\Delta t))] \Phi(i) m(i)+\widehat{p}_{i i} \hat{m}(i)
\end{aligned}
$$

Define

$$
\begin{aligned}
& \widehat{p}_{00}=1, \quad \widehat{p}_{0 i}=0 \text { for } i=1,2, \ldots, N, \\
& \widehat{p}_{i i}=0 \text { for } i=1,2, \ldots, N .
\end{aligned}
$$

Hence from (8.7), we obtain

$$
\widehat{m}(i)=E_{i}[\Phi(X(\Delta t))] \Phi(i) m(i) .
$$

For $i=1,2, \ldots, N$, if $E_{i} \Phi(X(\Delta t)) \neq 0$, we see from (8.5) that for all $j=$ $1,2, \ldots, N, j \neq i$,

$$
\widehat{p}_{i j}=\frac{q_{i j} \Phi(j)}{E_{i}[\Phi(X(\Delta t))]}=\frac{q_{i j} \Phi(j)}{\Sigma_{i \neq i} q_{i i} \Phi(t)} .
$$

For $i=1,2, \ldots, N$, if $E_{i}[\Phi(X(\Delta t))]=0$, we can define $\widehat{p}_{i j}, 1 \leqslant j \leqslant N, j \neq i$ arbitrarily such that

$$
\sum_{j=1}^{N} \hat{p}_{i j}=1 .
$$

From $(8.11)$ and $(8.12)$, we get

$$
\widehat{p}_{i 0}=0 \text { for all } i \neq 0 \text {. }
$$

In the following discussion, we suppose that

$$
E_{i}[\Phi(X(\Delta t))]>0 \text { for all } i=1,2, \ldots, N .
$$

Hence we have from (8.11) that for all $f \in H$,

$$
\hat{P} f(i)=\frac{E_{i}[(f \Phi)(X(\Delta t))]}{E_{i}[\Phi(X(\Delta t))]}
$$


Let $\left\{\hat{P}_{t} \mid t \in T\right\}$ be the semigroup generated by $\widehat{P}$. Then it is easy to see that $\left\{\widehat{P}^{\ell} \mid t \in T\right\}$ is subordinate to $\left\{Q^{t} \mid t \in T\right\}$. By using Theorem 7.1, there exists an IMF $M(\omega, t)$ of $X(\omega, t)$ generating $\left\{\hat{P}^{t} \mid t \in T\right\}$. From (7.15) in Section 7, we know that

$$
M(\omega, \Delta t)=\frac{\Phi(X(\omega, \Delta t))}{E_{X(\omega, 0)}^{\top}[\Phi(X((\Delta t))]} .
$$

Therefore, for all $k \in{ }^{*} \mathbf{N}$, we have

$$
M(\omega,(k+1) \Delta t)=\prod_{l=0}^{k} \frac{\Phi(\omega,(l+1) \Delta t)}{E_{X(\omega, l \Delta t)}[\Phi(X(\Delta t))]} .
$$

On the other hand, let $\Phi$ be an internal function in $H$ satisfying (8.14). We define $M(\omega, t)$ directly by $(8.17)$. Then we have the following

THEOREM 8.1. Assume that the hypotheses (8.I) and (8.14) hold. Then

(1) The semigroup $\left\{\left(Q^{\Phi}\right)^{t} \mid t \in T\right\}$ generated by $M(\omega, t)$ is symmetric with respect to the measure $E_{i}[\Phi(X(\Delta t))] \Phi(i) m(i)=\widehat{m}(i)$.

(2) $\mathcal{E}^{\Phi}(\cdot, \cdot)$ is the Dirichlet form associated with $\left\{\left(Q^{\Phi}\right)^{t} \mid t \in T\right\}$ and $\widehat{m}(\cdot)$.

Proof. Easy!

\section{Acknowledgement}

I have benefited a lot by the work under Prof. Sergio Albeverio at Ruhr-University Bochum, Germany, from April 1992 to July 1994. Moreover, I thank the very stimulating discussion with Prof. Tom Lindstrøm at University of Oslo, Norway, in 1993.The kind suggestion by the referees is appreciated for the improvement of both English and presentation of the paper. In particular, the reminding of the referees has led to the essential improvement of Lemma 3.8 and Theorem 3.3.

\section{References}

1. Albeverio, S. and Fan Ru-zong: Hyperfinite Dirichlet Forms ard Potential Theory, book in preparation (1996).

2. Albeverio, S., Fan Ru-zong, Röckner, $M$. and Stanual: 'A Remark on Coercive Forms and Associated Semigroups', In Partial Differential Equations and Mathematical Physics (Series: Operator Theory: Advances and Applications, Vol. 78, eds. M. Demuth and B.W. Schulze, Birkhäuser Verlag, Basel'Switzerland), 1995, 1-8.

3. Albeverio, S., Fenstad, J. E., Høegh-Krohn, R., and Lindstrøm, T: Nonstandard Methods in Stochastic Analysis and Mathematical Physics, Academic Press, New York, 1986.

4. Albeverio, S. and Ma Zhiming: Necessary and Sufficient Conditions for the Existence of mPerfect Processes Associated with Dirichlet Forms, Lecture Notes in Mathematics 1485, 374-406, Springer, Berlin, 1991.

5. Blumenthal, R. M. and Getoor, R. K.: Markov Processes and Potential Theory, Academic Press, New York, 1968. 
6. Fan Ru-zong: 'Nonstandard Construction of Symmetric Strong Markov Processes Associated with Dirichlet Forms', Proceeding of III Intemational Conference on Stochastic Processes, Geometry and Physics, Locamo, Switzerland, Eds. Sergio Albeverio et al. 247-277, 1995.

7. Fukushima, M.: Dirichlet Forms and Markov Processes, North-Holland, Amsterdam, Oxford, New York, 1980.

8. Fukushima, M. and Takeda, M.: 'A Transformation of Symmetric Markov Process and the Donsker-Varadhan Theory', Osaka J. Math. 21 (1984), 311-326.

9. Ma Zhiming and Röckner, M.: An Introduction to the Theory of Non-Symmetric Dirichlet Forms, Universitext, Springer-Verlag, 1992.

10. Meyer, P. A.: Probability and Potentials, Ginn Blaisdell, Massachusetts, 1966. 